\title{
A Novel Fuzzy Model Predictive Control of a Gas Turbine in the Combined Cycle Unit
}

\author{
Guolian Hou $\mathbb{D}^{1},{ }^{1}$ Linjuan Gong, ${ }^{1}$ Xiaoyan Dai, ${ }^{1}$ Mengyi Wang, ${ }^{1}$ and Congzhi Huang ${ }^{1,2}$ \\ ${ }^{1}$ School of Control and Computer Engineering, North China Electric Power University, Beijing 102206, China \\ ${ }^{2}$ Colleges and Universities Key Laboratory of Intelligent Integrated Automation, Guilin University of Electronic Technology, \\ Guangxi 541004, China
}

Correspondence should be addressed to Guolian Hou; hgl@ncepu.edu.cn

Received 11 July 2018; Accepted 8 September 2018; Published 5 November 2018

Guest Editor: Zhile Yang

Copyright (c) 2018 Guolian Hou et al. This is an open access article distributed under the Creative Commons Attribution License, which permits unrestricted use, distribution, and reproduction in any medium, provided the original work is properly cited.

The complex characteristics of the gas turbine in a combined cycle unit have brought great difficulties in its control process. Meanwhile, the increasing emphasis on the efficiency, safety, and cleanliness of the power generation process also makes it significantly important to put forward advanced control strategies to satisfy the desired control demands of the gas turbine system. Therefore, aiming at higher control performance of the gas turbine in the gas-steam combined cycle process, a novel fuzzy model predictive control (FMPC) strategy based on the fuzzy selection mechanism and simultaneous heat transfer search (SHTS) algorithm is presented in this paper. The objective function of rolling optimization in this novel FMPC consists of two parts which represent the state optimization and output optimization. In the weight coefficient selection of those two parts, the fuzzy selection mechanism is introduced to overcome the uncertainties existing in the system. Furthermore, on account of the rapidity of the control process, the SHTS algorithm is used to solve the optimization problem rather than the traditional quadratic programming method. The validity of the proposed method is confirmed through simulation experiments of the gas turbine in a combined power plant. The simulation results demonstrate the remarkable superiorities of the adopted algorithm with higher control precision and stronger disturbance rejection ability as well as less optimization time.

\section{Introduction}

At present, thermal power generation still occupies the leading position in the power generation industry in China $[1,2]$. Meanwhile, the increasing attention paid to energy conservation and emission reduction [3] has promoted the emergence and development of clean energy-based power generation. As a readily available clean energy, natural gas shows outstanding superiorities in environmental sustainability, energy efficiency, and security [4]. Therefore, there are certain development prospects using natural gas as fuel in power generation.

The combined cycle unit which consists of the gas turbine, heat recovery boiler, and steam turbine [5] is one of the maturely developed power generation devices using natural gas. In the generation process, the generator is driven by the gas turbine; meanwhile, turbine exhaust enters the heat recovery boiler and the residual heat of the gas is made full use of through the steam circulation system [6]. In this way, the characteristics of the gas turbine cycle with high average endothermic temperature and the steam turbine cycle with low average exothermic temperature are combined based on the principle of thermal cascade utilization. Considering the aforementioned process, the gas turbine is regarded as one of the most critical components in the combined cycle unit. Accordingly, it needs to be precisely controlled. However, because of the complex characteristics of the gas turbine with nonlinearity, variable coupling, and uncertainty, conventional control methods have difficulty in achieving the desired performance $[7,8]$. Thus, it is remarkably significant to select one of the advanced control strategies for the control of the gas turbine.

With the flourishing development of control methods in recent years, some advanced control algorithms have emerged continuously and obtained great achievements, such as fuzzy control [9-11], predictive control [12-15], 
and neural network control [16-19]. In all of those methods, predictive control is developed in the industrial process directly [20]. The predictive control consists of these three main components: predictive model, rolling optimization, and feedback correction [21]. Compared with the other aforementioned control strategies, predictive control has stronger ability to handle the time-varying processes with variable constraints and disturbances. Furthermore, this high-performance strategy also can combine the practical requirements of the control process with theoretical derivation maximally. Consequently, the predictive control will be the selected control method in this paper. Since its appearance, predictive control has attracted the great attention of experts and scholars of the world. Among all of the existing predictive control algorithms, the generalized predictive control (GPC) method [22, 23] and the model predictive control (MPC) method $[24,25]$ are commonly used. The most difference between these two predictive control strategies is that the GPC algorithm is based on the controlled autoregressive integrated moving average (CARIMA) model while the MPC method is based on the state space model [26]. In view of the state space model, it can not only reflect the internal state of the system but also reveal the relationship between the internal state and external input/output variables [27]. Therefore, the MPC algorithm will be used as the starting point of the novel algorithm adopted in this paper. In ElFerik et al. [28], a distributed nonlinear model predictive control (NMPC) method was put forward for formation control of constrained autonomous vehicles in consideration of the communication bandwidth limitation and transmission delays. The collision was avoided effectively through the spatially filtered potential field, and the satisfactory performance of the presented scheme was also demonstrated through simulation experiments in either strongly connected or weakly connected networks. However, the model uncertainty and disturbances were not taken into consideration which would cause the reduction of control performance and universality of the proposed algorithm. In addition to that, the model predictive control (MPC) algorithm was applied to the coalconveying process of a coal-fired power plant [29]. In that control scheme, the energy models of the belt conveyor and crusher were constructed and employed to formulate an open-loop energy efficiency optimization problem. Then, based on the open-loop optimization problem, a close-loop MPC strategy was adopted and applied to handle the nonlinearity of the controlled system. This control strategy showed strong robustness, adaptability, and energy-saving ability. Unfortunately, the performance reduction caused by the uncertainties of the system was inevitable. Considering the control of the complex nonlinear process with strong coupling and model mismatch, an extended nonminimal state space predictive control (ENMSSPC) strategy combined with a modified linear quadratic regulator (LQR) was put forward for the improvement of the control performance [30]. Although this method showed better properties with ideal tracking performance and strong disturbance rejection ability, the accompanying increased calculation burden was ignored at the same time. Furthermore, the distributed stochastic MPC [31, 32] was adopted for these control systems with uncertainty and probabilistic constraints as well as disturbances. According to the distribution manner, only one subsystem was selected at each time interval. Meanwhile, the probabilistic constraints were transformed into a set of deterministic constraints through the probabilistic distribution. The outstanding advantages of this strategy with assurances of recursive feasibility and close-loop stability for any updated sequence had been verified through numerical experiments. Nevertheless, the rapidity of the control process should also be taken into account in the design of the controller.

Considering that there are many values that cannot be expressed accurately in most control processes, the concept of "fuzzy" was introduced and applied in the construction of the control strategy to handle uncertainties existing in the controlled system [33, 34]. Aiming at a better dynamic response and reduction in the low-frequency ripple of integrated LED drivers, a fast fuzzy logic control algorithm based on a Takagi-Sugeno-Kang fuzzy controller was put forward [35]. The high-speed mathematical engine of a digital signal controller was used to increase the calculation speed which contributed to a less settling time of the control process. Unfortunately, this method had a less positive effect on the improvement of control precision. The accurate mobile positioning in the cellular network is a matter of great challenge when it comes to the uncertainty in the signal intensity measurement process. Therefore, the fuzzy reasoning was added to a Kalman filter for improving the accuracy of localization [36], and the formed fuzzy extended filter showed remarkable feasibility and superiority compared with single Kalman filter method or fuzzy control strategy [37]. What is more, the fuzzy logic also can be satisfactorily combined with the MPC algorithm. In Boulkaibet et al. [38], the T-S fuzzy model of each typical operating condition was identified by particle swarm optimization (PSO) clustering method and Kernel ridge regression algorithm initially. Then, the predictive control was introduced based on the fuzzy model, and the resulting adaptive fuzzy predictive control strategy could deal with the disturbances and parameter variations effectively. Nevertheless, the output variations and operating time of the control process were not taken into account. In view of the nonlinearities and various disturbances inevitably existing in a complex multivariable system, the fuzzy model predictive control (FMPC) method has become a desired candidate for the promotion of the control performance [39]. The application of extended state observer-based FMPC algorithms on the boiler-turbine system in the ultrasupercritical unit of the power plant had handled these problems and obtained better tracking ability [40], whereas the rapidity of this method needed to be verified. Thus, considering the complex characteristics of the controlled system and the significant superiorities of the FMPC mentioned above, a new FMPC strategy will be constructed for the control of the gas turbine system in a combined cycle unit.

As one of the emerging intelligent algorithms in recent years, the bionic algorithm has developed rapidly with many branches, in which the swarm-based optimization technique $[41,42]$ stands out and has been extensively applied in modeling and control processes. Model identification of an 
unknown plant is important for the subsequent controller design to meet desired control specifications. Therefore, in the modeling process of an unknown digital system, a multiple subpopulation-based modified particle swarm optimization (PSO) algorithm was introduced to solve the coefficients of the infinite impulse response (IIR) digital filter [43]. Although this method was able to overcome the multimodal error surface problem effectively, its precise identification would decrease when the order of the controlled system has increased. In addition, an artificial bee colony (ABC) algorithm and a grey wolf optimization (GWO) algorithm were used for solving portfolio optimization problems with cardinality constrains [44] and smart wind speed forecasting [45], respectively. The solution approach combined with the $A B C$ algorithm had reduced the computational difficulties from large-scale optimization problems to a certain extent. Meanwhile, the proposed new hybrid strategy with the addition of GWO algorithm had ensured high accuracy and stability in the prediction process of wind speed. However, similar to most other swarm intelligence algorithms, it is difficult to achieve a win-win situation considering both the accuracy and the rapidity of the control process. Heat transfer search (HTS) algorithm is a novel method for solving the optimization problem which was presented in 2015 [46]. On the basis of the law of thermodynamics in the heat transfer process, the molecule which consisted of the decision variables of the optimization problem made every effort to maintain the thermal equilibrium between itself and the surrounding environment through the three manners of heat transfer: conduction, convection, and radiation. This algorithm had won the first place in the Friedman rank test compared with some other optimization methods, such as PSO algorithm, ABC algorithm, and Yin-Yang pair optimization. Furthermore, in view of the expensive calculating burden in the iterative optimization process, a simultaneous heat transfer search (SHTS) was adopted and its lower computational complexity had been verified through simulation experiments [47]. Consequently, a SHTS algorithm considering the variable constraints will be utilized in this paper to decrease the computational complexity and optimization time of the control process.

On account of the content mentioned above, a novel fuzzy model predictive control (FMPC) which combined fuzzy selection mechanism with the SHTS algorithm is proposed for the promotion of control performance. Firstly, the objective function of the presented algorithm is made up of two parts which represent the state optimization and output optimization. These two parts occupy different weights according to the variations of state variables and output error of the system. Then, considering the uncertainties of the control system, the fuzzy selection mechanism is introduced for the adaptive selections of the above two weighting coefficients in each time interval. What is more, aiming at the reductions of computational burden and optimization time of the control process, the quadratic programming solving process in rolling optimization of conventional MPC is replaced by a SHTS algorithm while taking the variable constraints into account. This SHTS method is a less subjective strategy with two artificially defined parameters. Unlike the original HTS method, the whole population of SHTS is divided into three groups randomly, and each group is assigned to one of three modes of heat transfer. In this way, a great decline of the optimization time of the control process is obtained easily. Finally, the proposed algorithm will be utilized in the control of the gas turbine system in the combined cycle unit and compared with some other similar algorithms. The main contributions of this paper are summarized as follows briefly:

(i) A novel FMPC algorithm is put forward based on the fuzzy selection of parameters while taking the state optimization problem and output optimization problem into consideration simultaneously. The addition of the fuzzy selection mechanism can overcome the uncertainties of the complex control system effectively. Furthermore, the precision of the control process also has great promotion

(ii) Based on the FMPC algorithm described in (i), a HSTS method which takes variable constraints into account is adopted to replace the quadratic programming solving process in rolling optimization of the traditional MPC algorithm. This replacement can decrease the optimization time of the control system to a certain extent

(iii) The presented algorithm is firstly applied in the control of the gas turbine in a combined cycle unit. The simulation results demonstrate that this control strategy can overcome the complex characteristics of a multivariable system effectively. Meanwhile, it is proved to be valuable in the promotions of control precision, disturbance rejection ability, and optimization speed.

The rest of paper is organized as follows. The gas turbine system in the combined cycle unit is described briefly in Section 2. The construction process of the proposed novel FMPC algorithm is introduced in Section 3. Next, the simulation results are shown to verify the superiorities of this algorithm in Section 4. Finally, some general conclusions are presented in Section 5.

\section{Problem Description}

2.1. The Combined Cycle Unit in a Power Plant. In order to increase the overall efficiency of electric power plants, multiple thermodynamic cycles can be combined to fully utilize energy. The term "combined cycle" refers to the combination of different thermodynamic processes. Compared with the simple cycle, the combined cycle can achieve the utmost utilization of energy and produce more electricity from the same fuel.

The combined cycle is always assembled by a topping cycle and a bottoming cycle, and its most common type is combined with the Brayton cycle operated by a gas turbine and the Rankine cycle operated by a steam turbine separately, which is usually called the combined cycle gas turbine (CCGT). The principle of the combined cycle is that the 


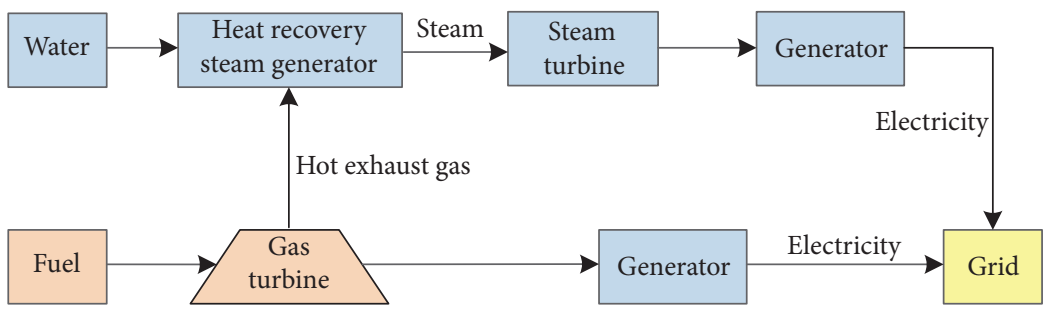

Figure 1: Combined cycle schematic diagram.

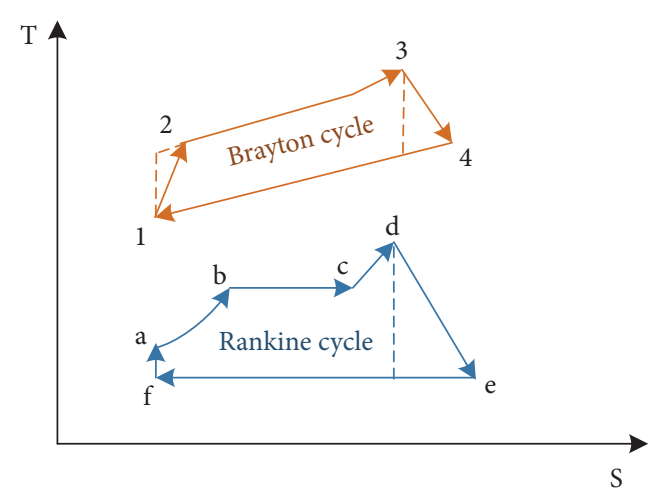

FIGURE 2: Combined cycle temperature-entropy diagram (dashed line: theoretical cycle process; solid line: real cycle process).

temperature of the working fluid is still high enough to supply power for the subsequent engine after completing the cycle in the first engine. The operating process of the combined cycle can be described briefly as follows. The gas is burned in a gas turbine and becomes exhaust gases with temperature range from $550^{\circ} \mathrm{C}$ to $610^{\circ} \mathrm{C}$. Meanwhile, the heat obtained in the burning process is converted to electric power through a coupled generator. Then a heat recovery steam generator is employed to produce steam and hot water by capturing exhaust heat. Next, a steam turbine is driven by the steam to deliver additional electricity by driving the corresponding generator. Finally, these two electric powers are transported to the grid synchronously. The diagram of the combined cycle unit described above is structured in Figure 1.

The basic combined cycle unit consists of two cycles as shown in Figure 2: the cycle 1-2-3-4-1 depicts the heat transfer process existing in the high temperature region while the cycle a-b-c-d-e-f-a represents the Rankine steam cycle existing in the lower temperature region. In addition, the heat recovery steam generator captures the exhaust energy to heat water into steam and deliver it to the steam turbine. Furthermore, the steam turbine transfers its energy to the generator, where the energy is converted into additional electricity.

2.2. Description of the Gas Turbine System. The efficiency of the combined cycle gas turbine depends on the performance of the gas turbine which is regarded as the core equipment of the combined cycle power plant. However, the existing conventional control strategies cannot deal with the complex characteristics of the gas turbine effectively. As a result, it is necessary to develop advanced control schemes based on the dynamic characteristics of the gas turbine to meet grid dispatch requirements quickly.

There are three main components of the gas turbine system diagram which are depicted briefly in Figure 3: compressor, combustion chamber, and turbine. First, the air is compressed by the gas turbine and mixed with fuel. Then, the mixture is heated to a high temperature and high pressure gas to move through the turbine blades and make the turbine spin. Finally, a generator is driven by the mechanical energy from high-speed rotation of the turbine to produce electricity.

High generating efficiency and stable rotor speed, as well as the ideal exhaust temperature and exhaust flow are main control objectives of a gas turbine system. On the one hand, the turbine inlet temperature cannot be too high when it is limited by blade thermal stress. On the other hand, if this temperature decreases excessively, the efficiency of the combined cycle power plant is also reduced. Therefore, turbine inlet temperature should be kept as close as possible to the ideal value. What is more, to ensure the efficiency of the power plant, not only the fuel flow but also the inlet guide vane of the gas turbine should be adjusted to maintain a certain fuel-air ratio when the load of the combined cycle unit has changed.

Considering the most significant factors during the operating process, the gas turbine system can be simplified as a system with two input variables and four output variables as illustrated in Figure 4.

In Figure $4, G_{f}$ and IGV are two input variables which represent the fuel flow and inlet guide vane, respectively; in addition, the output variables $N_{\mathrm{op}}, n_{\mathrm{rs}}, T_{\mathrm{ex}}$, and $G_{\mathrm{ex}}$ stand for output power, rotor speed, exhaust temperature, and exhaust flow, respectively. Although this simplified multivariable model can reveal the main features of the gas turbine, obviously, its complex characteristics with nonlinearity, uncertainties, and coupling are also difficulties in the controller design of the gas turbine system. Therefore, it is remarkably significant to make further improvement of the original control strategy to satisfy the aforementioned control objectives of the gas turbine system in a combined cycle unit.

\section{Simultaneous Heat Transfer Search-Based Fuzzy Model Predictive Control}

The local linear models of the gas turbine system can be obtained through the modeling method proposed in Hou et al. [48] which overcomes the nonlinearity of the complex 


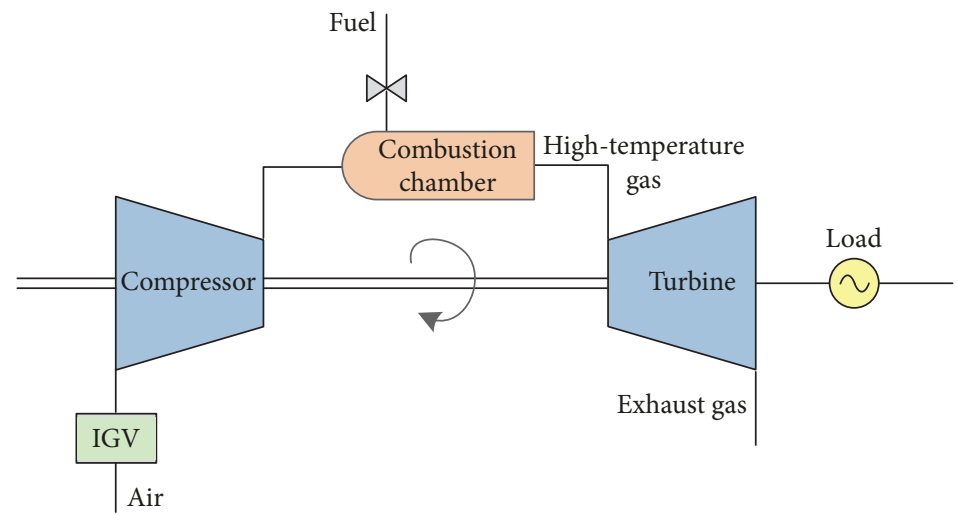

FIGURE 3: Gas turbine system diagram.

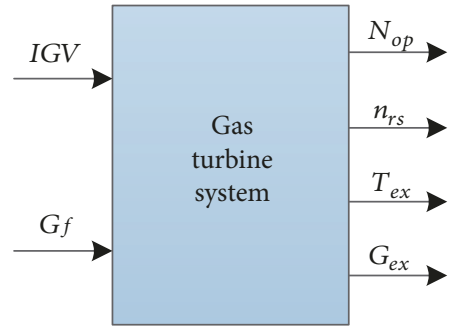

FIgURE 4: Simplified gas turbine model diagram.

system effectively. Therefore, on the basis of the identified model, a novel simultaneous heat transfer search- (SHTS-) based fuzzy model predictive control (FMPC) algorithm is adopted in this part. Firstly, a novel FMPC strategy is constructed to improve the control precision while considering the uncertainties in the control system. Then, the SHTS optimization method is introduced for reducing optimization time.

3.1. The Novel FMPC Algorithm. The proposed FMPC algorithm is an improvement of the original MPC combined with fuzzy selection mechanism; it mainly has the structure shown in Figure 5.

In the diagram shown above, $r(k)$ is the set point of the $k$-th time interval, $u(k)$ is the input variable vector of the controlled process while $y(k)$ is the output variable vector, and $\hat{x}(k)$ is the predictive state variable vector. Furthermore, $\hat{y}(k+j)$ stands for the predictive output of the $j$-th steps later after the $k$-th time interval, $e(k)$ is the error vector between predictive outputs and actual outputs, $c_{1}$ and $c_{2}$ are the weight coefficients of output optimization and state optimization, respectively, obtained by fuzzy reasoning from fuzzy selection mechanism.

A multivariable system with $m$ input variables, $n$ state variables, and $q$ output variables can be expressed as follows:

$$
\begin{aligned}
x(k+1)= & A x(k)+B u(k), \\
y(k)= & C x(k), \\
& k=1, \ldots, N,
\end{aligned}
$$

where $N$ is the sampling time, $x(k)=\left[x_{1}(k), x_{2}(k), \ldots\right.$, $\left.x_{n}(k)\right]^{T}$ is the state vector and $u(k)=\left[u_{1}(k), u_{2}(k), \ldots\right.$, $\left.u_{m}(k)\right]^{T}$ and $y(k)=\left[y_{1}(k), y_{2}(k), \ldots, y_{q}(k)\right]^{T}$ are the input variable vector and output variable vector, respectively; meanwhile, matrices $A, B$, and $C$ represent the state matrix, input matrix, and output matrix, respectively, of the system.

Considering that $\Delta x(k)=x(k)-x(k-1)$ and $\Delta u(k)=$ $u(k)-u(k-1)$, the increment vector of the output variables is exhibited as

$$
\Delta y(k+1)=C A \Delta x(k)+C B \Delta u(k), \quad k=1, \ldots, N .
$$

Constructing a new state variable vector as $\bar{x}(k)=$ $[\Delta x(k) y(k)]^{T}$, the corresponding state space model shown as (3) can be obtained.

$$
\begin{gathered}
\bar{x}(k+1)=\bar{A} \bar{x}(k)+\bar{B}(k) \Delta u(k), \\
\hat{y}(k)=\bar{C} \bar{x}(k), \\
k=1, \ldots, N,
\end{gathered}
$$

where

$$
\begin{aligned}
& \bar{A}=\left[\begin{array}{cc}
A & 0 \\
C A & I
\end{array}\right], \\
& \bar{B}=\left[\begin{array}{c}
B \\
C B
\end{array}\right], \\
& \bar{C}=\left[\begin{array}{ll}
0 & I
\end{array}\right] .
\end{aligned}
$$

Set the predictive horizon as $P$ while the control horizon as $M$, then define the state vector $\bar{X}$, control vector $\Delta U$, and predictive output vector $\widehat{Y}$ in future $P$ steps as 


$$
\begin{aligned}
& \bar{X}(k)=\left[\begin{array}{c}
\bar{x}(k+1) \\
\bar{x}(k+2) \\
\vdots \\
\bar{x}(k+P)
\end{array}\right], \\
& \Delta U(k)= {\left[\begin{array}{c}
\Delta u(k) \\
\Delta u(k+1) \\
\vdots \\
\Delta u(k+M-1)
\end{array}\right], } \\
& \widehat{Y}(k)=\left[\begin{array}{c}
\hat{y}(k+1) \\
\hat{y}(k+2) \\
\vdots \\
\hat{y}(k+P)
\end{array}\right] .
\end{aligned}
$$

Next, the $\bar{X}, \Delta U$, and $\widehat{Y}$ can be calculated through

$$
\begin{gathered}
\bar{X}(k)=F_{x} \bar{x}(k)+\Gamma_{x} \Delta U(k), \\
\widehat{Y}(k)=F_{y} \bar{x}(k)+\Gamma_{y} \Delta U(k), \\
U(k)=l u(k-1)+L \Delta U(k), \\
k=1, \ldots, N,
\end{gathered}
$$

where

$$
\begin{aligned}
\Gamma_{x} & =\left[\begin{array}{ccccc}
\bar{B} & 0 & 0 & \ldots & 0 \\
\bar{A} \bar{B} & \bar{B} & 0 & \ldots & 0 \\
\bar{A}^{2} \bar{B} & \bar{A} \bar{B} & \bar{B} & \ldots & 0 \\
\vdots & \vdots & \vdots & \ddots & \vdots \\
\bar{A}^{P-1} \bar{B} & \bar{A}^{P-2} \bar{B} & \bar{A}^{P-3} \bar{B} & \ldots & \bar{A}^{P-M} \bar{B}
\end{array}\right], \\
\Gamma_{y} & =\left[\begin{array}{ccccc}
\bar{C} \bar{B} & 0 & 0 & \ldots & 0 \\
\bar{C} \bar{A} \bar{B} & \bar{C} \bar{B} & 0 & \ldots & 0 \\
\bar{C} \bar{A}^{2} \bar{B} & \bar{C} \bar{A} \bar{B} & \bar{C} \bar{B} & \ldots & 0 \\
\vdots & \vdots & \vdots & \ddots & \vdots \\
\bar{C} \bar{A}^{P-1} \bar{B} & \bar{C} \bar{A}^{P-2} \bar{B} & \bar{C} \bar{A}^{P-3} \bar{B} & \ldots & \bar{C}^{P-M} \bar{B}
\end{array}\right], \\
F_{x} & =\left[\begin{array}{c}
\bar{A} \\
\bar{A}^{2} \\
\vdots \\
\bar{A}^{P}
\end{array}\right], \\
F_{y} & =\left[\begin{array}{c}
\bar{C}_{\bar{A}} \\
\bar{C} \bar{A}^{2} \\
\vdots \\
\bar{C} \bar{A}^{P}
\end{array}\right],
\end{aligned}
$$

$$
\begin{gathered}
l=\left[\begin{array}{c}
1 \\
1 \\
\vdots \\
1
\end{array}\right], \\
L=\left[\begin{array}{cccc}
1 & 0 & \cdots & 0 \\
1 & 1 & \cdots & 0 \\
\vdots & \vdots & \ddots & \vdots \\
1 & 1 & \cdots & 1
\end{array}\right] .
\end{gathered}
$$

Then, the objective function should be structured and minimized to obtain the control increment $\Delta U$.

In view of the output optimization problem, the objective function has the following expression.

$$
J_{1}(k)=\|W(k)-\widehat{Y}(k)\|_{Q_{y}}^{2}+\|\Delta U(k)\|_{R_{y}}^{2}, \quad k=1, \ldots, N,
$$

in which, $W(k)$ is the reference trajectory of the system and $Q_{y}$ is the error weight coefficient while $R_{y}$ is the control weight coefficient of output optimization.

Furthermore, the state optimization-based objective function can be defined as

$$
J_{2}(k)=\|\bar{X}(k)\|_{Q_{x}}^{2}+\|\Delta U(k)\|_{R_{x}}^{2}, \quad k=1, \ldots, N,
$$

where $Q_{x}$ is the state weight coefficient and $R_{x}$ is the control weight coefficient of state optimization.

Substituting (6) into (8) and (9), we omit items that are not related to the control effect. The simplified objective functions are formulated as

$$
\begin{aligned}
J_{1}(k)= & \Delta U^{T}(k)\left(\Gamma_{y}^{T} Q_{y} \Gamma_{y}+R_{y}\right) \Delta U(k) \\
& +2 \Gamma_{y}^{T} Q_{y}\left[F_{y} \bar{x}(k)-W(k)\right] \Delta U(k), \\
J_{2}(k)= & \Delta U^{T}(k)\left(\Gamma_{x}^{T} Q_{x} \Gamma_{x}+R_{x}\right) \Delta U(k) \\
& +2 \Gamma_{x}^{T} Q_{x} F_{x} \bar{x}(k) \Delta U(k) .
\end{aligned}
$$

Next, on account of constraints of control variables, a new objective function $J$ will be constituted based on the subfunctions $J_{1}(k)$ and $J_{2}(k)$.

$$
\begin{aligned}
J & =c_{1} J_{1}+c_{2} J_{2}=\Delta U^{T}(k) H \Delta U(k)+2 G \Delta U(k), \\
\text { s.t. } \quad U_{\min } & \leq U(k) \leq U_{\max }, \\
\Delta U_{\min } & \leq \Delta U(k) \leq \Delta U_{\max },
\end{aligned}
$$




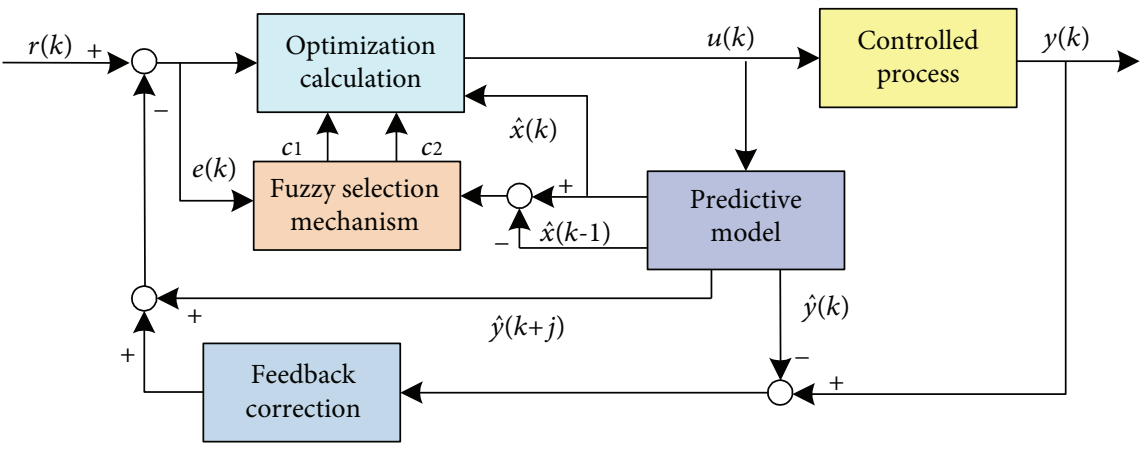

FIgURE 5: Simplified diagram of proposed FMPC strategy.

where

$$
\begin{aligned}
& H=c_{1}\left(\Gamma_{y}^{T} Q_{y} \Gamma_{y}+R_{y}\right)+c_{2}\left(\Gamma_{x}^{T} Q_{x} \Gamma_{x}+R_{x}\right), \\
& G=c_{1} \Gamma_{y}^{T} Q_{y}\left[F_{y} \bar{x}(k)-W(k)\right]+c_{2} \Gamma_{x}^{T} Q_{x} F_{x} \bar{x}(k) .
\end{aligned}
$$

$c_{1}$ and $c_{2}$ represent the weight coefficients of $J_{1}$ and $J_{2}$, respectively.

Consequently, on the basis of objective function (11), the following control law of presented FMPC can be achieved by calculating the partial derivation of $\Delta U$.

$$
\begin{aligned}
\Delta U(k)= & {\left[c_{1}\left(\Gamma_{y}^{T} Q_{y} \Gamma_{y}+R_{y}\right)+c_{2}\left(\Gamma_{x}^{T} Q_{x} \Gamma_{x}+R_{x}\right)\right]^{-1} } \\
& \cdot\left\{c_{1} \Gamma_{y}^{T} Q_{y}\left[W(k)-F_{y} \bar{x}(k)\right]-c_{2} \Gamma_{x}^{T} Q_{x} F_{x} \bar{x}(k)\right\} .
\end{aligned}
$$

The first step of the control law will be used in the control system in each time interval. Considering the parameter uncertainties existing in the controlled system and inherent fuzzy properties of variables, the fuzzy selection mechanism described in the next part is introduced in the selection process of weight coefficients $c_{1}$ and $c_{2}$.

3.2. Fuzzy Selection of the Weight Coefficients. In the fuzzy selection mechanism of weight coefficients adopted in this part, the sum of absolute value of system output error $e_{i}(k)$ $(i=1, \ldots, q, k=1, \ldots, N)$ and the sum of absolute value of state variable variation $\Delta x_{i}(k)(i=1, \ldots, q, k=1, \ldots, N)$ shown in (14) are taken as inputs of fuzzy rules while $c_{1}$ and $c_{2}$ are regarded as corresponding outputs.

$$
\begin{gathered}
E(k)=\sum_{i=1}^{q}\left|e_{i}(k)\right|, \\
\Delta X(k)=\sum_{i=1}^{n}\left|\Delta x_{i}(k)\right|, \\
\quad k=1, \ldots, N,
\end{gathered}
$$

where $e_{i}(k)=y_{i}(k)-\hat{y}_{i}(k)(i=1, \ldots, q)$ and $\Delta x_{i}(k)=x_{i}(k)$ $-x_{i}(k-1)(i=1, \ldots, n)$.

Set the fuzzy domains of $E(k)$ and $\Delta X(k)$ as $[0,7]$ and $[0,100]$, respectively. Meanwhile, the domains of fuzzy outputs $c_{1}$ and $c_{2}$ are both assigned as $[0,1]$. In addition, all of the membership functions of inputs and outputs are defined as Gaussian functions for its ideal properties and the simplicity of the calculation process, and the shown seven fuzzy degrees are used in the description of each variable.

$$
S=\{\mathrm{NB}, \mathrm{NM}, \mathrm{NS}, \mathrm{ZO}, \mathrm{PS}, \mathrm{PM}, \mathrm{PB}\} .
$$

Then, membership function curves of input variables in this fuzzy selection system can be drawn as Figure 6 . The Gaussian function curves of the output variables are equally available.

In the weight coefficient selection process, the value of $c_{1}$ will be enlarged when the sum of output errors of the controlled system is increased; at the same time, the value of $c_{2}$ will be promoted when the sum of state variable variations is increased. Combining this basic regulation principle with expert experiences, the resulting fuzzy rules are illustrated as

$$
\begin{gathered}
\text { If } E=\mathrm{NB} \text { and } \Delta X=\mathrm{NB} \text {, then } c_{1}=\mathrm{PM} \text { and } c_{2}=\mathrm{NM} \text {, } \\
\vdots \\
\text { If } E=\mathrm{PB} \text { and } \Delta X=\mathrm{PB} \text {, then } c_{1}=\mathrm{PB} \text { and } c_{2}=\mathrm{ZO} .
\end{gathered}
$$

In order to ensure the integrity of the fuzzy rules, all of the rules are listed in following Table 1.

Consequently, the weight factors of output optimization and state optimization can be chosen reasonably when given the inputs of fuzzy rules. However, this parameter selection process will increase the settle time of the whole control system. Under this circumstance, a SHTS algorithm will be presented for the replacement of the conventional quadratic programming solving method to reduce the optimization time.

3.3. Simultaneous Heat Transfer Search Algorithm in Optimization Process. In order to solve a certain optimization problem through the quadratic programming, an objective function with standard quadratic programming form needs to be structured. Nevertheless, this procedure has increased the solving complexity to a great extent. On the contrary, the standardization step is not necessary in the optimization process of the simultaneous heat transfer search (SHTS) 

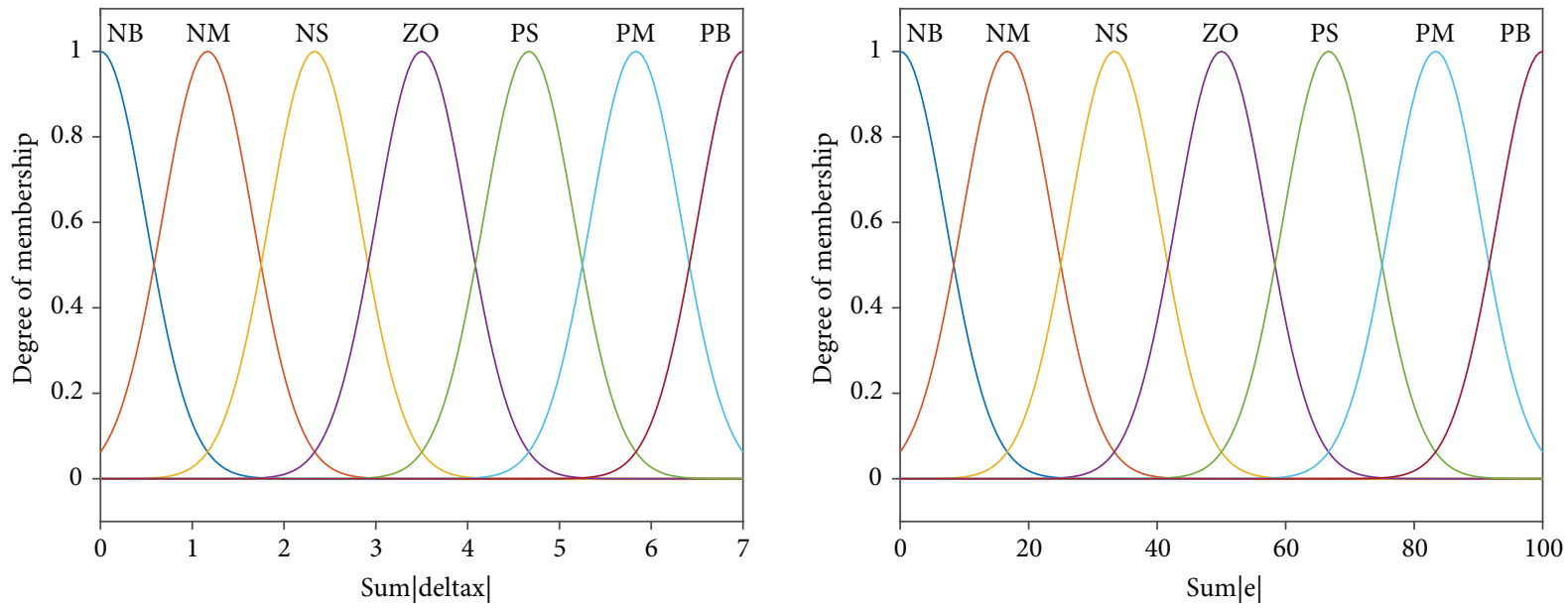

FIgURE 6: Gaussian membership function curves of fuzzy input variables.

TABLE 1: Fuzzy rules of weight coefficients selection process.

(a)

\begin{tabular}{ccccccccc}
\hline$c 1$ & & & & \multicolumn{1}{c}{$E$} \\
& & PB & PM & PS & ZO & NS & NM & NB \\
& PB & PB & PM & PS & ZO & NS & NM & NB \\
& PM & PB & PM & PS & ZO & ZO & NS & NS \\
& PS & PB & PM & PM & ZO & ZO & ZO & NS \\
& ZO & PB & PM & PM & PS & ZO & ZO & ZO \\
& NS & PB & PB & PM & PM & PS & PS & ZO \\
& NM & PB & PB & PB & PM & PM & PM & PS \\
& NB & PB & PB & PB & PB & PB & PB & PM \\
\hline
\end{tabular}

(b)

\begin{tabular}{|c|c|c|c|c|c|c|c|c|}
\hline \multirow{2}{*}{$c 2$} & & \multicolumn{7}{|c|}{$F$} \\
\hline & & $\mathrm{PB}$ & $\mathrm{PM}$ & PS & $\mathrm{ZO}$ & NS & $\mathrm{NM}$ & $\mathrm{NB}$ \\
\hline \multirow{7}{*}{$\Delta X$} & $\mathrm{~PB}$ & $\mathrm{ZO}$ & PS & $\mathrm{PM}$ & $\mathrm{PM}$ & $\mathrm{PM}$ & $\mathrm{PB}$ & $\mathrm{PB}$ \\
\hline & $\mathrm{PM}$ & $\mathrm{ZO}$ & PS & PS & $\mathrm{PM}$ & $\mathrm{PM}$ & $\mathrm{PM}$ & $\mathrm{PB}$ \\
\hline & PS & NS & $\mathrm{ZO}$ & NS & PS & PS & $\mathrm{PM}$ & $\mathrm{PM}$ \\
\hline & $\mathrm{ZO}$ & NM & NS & NM & NS & NS & PS & $\mathrm{PM}$ \\
\hline & NS & NB & $\mathrm{NM}$ & NM & $\mathrm{NM}$ & NM & $\mathrm{ZO}$ & $\mathrm{ZO}$ \\
\hline & NM & NB & NB & NB & NM & NM & NS & NS \\
\hline & NB & NB & NB & NB & $\mathrm{NB}$ & NB & $\mathrm{NM}$ & NM \\
\hline
\end{tabular}

algorithm mentioned. Therefore, the SHTS algorithm will be applied in every iterative optimization process to realize the promotions of optimization speed and control performance. The main steps of the SHTS method are summarized as follows.

Step 1. Initialize the population size NP, search dimension $D$ and maximum iteration number $K$, as well as the upper bound $\Delta U_{\max }$ and lower bound $\Delta U_{\min }$ of the variable vector to be optimized, in other words, the control variable vector $\Delta U(k)(k=1, \ldots, N)$ mentioned above.

Step 2. Generate the initial population $\Delta U(k)$ with size of $N$ $P \times D$ through

$$
\Delta U(k)=\Delta U_{\min }+r\left(\Delta U_{\max }-\Delta U_{\min }\right), \quad k=1, \ldots, N
$$

where $r$ is a random $N P \times D$ matrix with values between 0 and 1.

Step 3. Calculate the value of fitness function $J(k)$, in other words, the aforementioned objective function, based on the initial population. Then, select the member with minimum fitness as the best one and denote as $\left(\Delta U_{\text {best }}(k), J_{\text {best }}(k)\right)$ $(k=1, \ldots, N)$.

Step 4. Divide the population into three equal parts randomly and distribute each part to one of the three heat transfer modes. Furthermore, denote the subpopulations with conduction phase, convection phase, and radiation phase as $\Delta U_{D}(k), \Delta U_{V}(k)$, and $\Delta U_{R}(k)$, respectively.

Step 5. Implement following three heat transfer modes of each subpopulation.

Conduction phase: obtain new population through the equation described as

$$
\Delta U_{D}^{*}(k)=\Delta U_{R}(k)-r_{D} \Delta U_{R}(k), \quad k=1, \ldots, N,
$$

where $r_{D}$ is a random $(\mathrm{NP} / 3) \times D$ matrix with values between 0 and 1 .

Convection phase: define $c(k)=r_{V} \Delta U_{\text {best }}(k) R$, in which $r_{V}$ is a random matrix of $1 \times D$ dimension with values between 0 and 1 and $R$ is a matrix of $1 \times D$ dimension with values between 1 and 2 . Then, generate a new matrix $\mathrm{Cc}(k)$ which consists of $(\mathrm{NP} / 3)$ copies of $c(k)$; therefore, the size of matrix $\mathrm{Cc}(k)$ is $(\mathrm{NP} / 3) \times D$. Then, obtain the new population through. 


$$
\Delta U_{V}^{*}(k)=\Delta U_{V}(k)+\operatorname{Cc}(k), \quad k=1, \ldots, N .
$$

Radiation phase: randomly divide the subpopulation $\Delta U_{R}(k)$ into two equal parts $\Delta U_{R 1}(k)$ and $\Delta U_{R 2}(k)$. Next, assign every member of these two parts to a partner which is selected from $\Delta U_{R}(k)$ and denote the partner populations as $\Delta U_{R 1}^{\prime}(k)$ and $\Delta U_{R 2}^{\prime}(k)$, respectively. Then, generate the new population determined as

$$
\begin{gathered}
\Delta U_{R 1}^{*}(k)=\Delta U_{R 1}(k)-r_{R 1}\left(\Delta U_{R 1}(k)-\Delta U_{R 1}^{\prime}(k)\right), \\
\Delta U_{R 2}^{*}(k)=\Delta U_{R 2}(k)-r_{R 2}\left(\Delta U_{R 2}(k)-\Delta U_{R 2}^{\prime}(k)\right), \\
k=1, \ldots, N,
\end{gathered}
$$

where $r_{R 1}$ and $r_{R 2}$ are both the random matrices of the $(\mathrm{NP} / 6) \times D$ dimension with values between 0 and 1 .

Step 6. Construct the new population $\Delta U^{*}(k)=\left\{\Delta U_{D}^{*}(k)\right.$, $\left.\Delta U_{V}^{*}(k), \Delta U_{R 1}^{*}(k), \Delta U_{R 2}^{*}(k)\right\}$ based on the combination of $\Delta U_{D}^{*}(k), \Delta U_{V}^{*}(k), \Delta U_{R 1}^{*}(k)$, and $\Delta U_{R 2}^{*}(k)$.

Step 7. Modify the new population through (21) considering its feasibility in the search region.

$$
\begin{array}{r}
\Delta U^{*}(k)=\min \left(\Delta U^{*}(k), \Delta U_{\max }\right), \\
\Delta U^{*}(k)=\max \left(\Delta U^{*}(k), \Delta U_{\min }\right), \\
k=1, \ldots, N .
\end{array}
$$

Step 8. Calculate the fitness $J^{*}(k)$ of the new population and make a greedy choice with the initial optimal fitness $J_{\text {best }}(k)$. If the new fitness $J^{*}(k)$ is smaller than the original $J_{\text {best }}(k)$, the corresponding new population $\Delta U^{*}(k)$ will be selected as the updated optimum solution $\Delta U_{\text {best }}$ $(k)$ and $J_{\text {best }}(k)=\min \left(J^{*}(k), J_{\text {best }}(k)\right)$, else, the new population will be abandoned and search for another population will begin.

Step 9. If the control precision has satisfied the ideal demands or the number of iterations has reached the limitation, end this optimization process and apply the first step of $\Delta U_{\text {best }}$ $(k)$ to the controller. Then, start a new optimization process. Else, go back to step 2 .

The above discussed optimization process combined with SHTS can be simplified as Figure 7.

\section{Illustrative Example}

The remarkable advantages of the proposed control strategy should be demonstrated through illustrative examples to prove its feasibility and practicability. Therefore, the model of the gas turbine system of a combined cycle unit in Taiyanggong power plant which was identified through the T-S fuzzy modeling [48] is introduced as (22) to verify the performance of the adopted algorithm.

$$
\begin{aligned}
x(k+1)= & {\left[\begin{array}{cccccccc}
0.7108 & 0.1329 & 0.0118 & 0.1484 & -0.0146 & -0.1158 & -0.0027 & -0.0227 \\
-0.1489 & 0.9345 & 0.0002 & -0.0620 & 0.0090 & 0.0115 & -0.0428 & -0.1032 \\
0.0746 & -0.0043 & 0.9400 & -0.0941 & -0.0195 & 0.0741 & -0.1209 & -0.0988 \\
-0.2143 & -0.1906 & 0.0309 & -0.1159 & 0.1166 & 0.1635 & -0.4621 & -0.2520 \\
0.0286 & 0.0147 & -0.0625 & -0.3999 & 0.6278 & 0.3467 & 0.2930 & -0.3420 \\
0.0373 & -0.0923 & -0.0704 & -0.3024 & -0.5453 & 0.6718 & 0.3564 & 0.0141 \\
-0.0817 & -0.0243 & -0.0668 & 0.1916 & -0.3849 & -0.1626 & 0.3355 & -0.5698 \\
0.0030 & 0.0077 & 0.0427 & -0.1866 & 0.0161 & 0.2533 & -0.4076 & 0.4210
\end{array}\right] x(k) } \\
& +\left[\begin{array}{ccccccccc}
-0.0893 & 0.0286 & 0.0424 & 0.5522 & 0.3725 & 0.4867 & -0.0914 & 0.0090 \\
-0.0294 & 0.0615 & -0.0016 & 0.5262 & 0.2295 & 0.3082 & 0.0382 & 0.1785
\end{array}\right]^{T} u(k), \\
y(k)= & {\left[\begin{array}{cccccccc}
4.4627 & -5.4469 & -11.5426 & 1.6233 & -0.9642 & 3.0510 & -4.0003 & 1.8654 \\
-14.6745 & 19.4560 & -22.0502 & -0.3933 & 4.9559 & -5.7160 & 3.4771 & -7.5768 \\
-19.5587 & -7.9487 & 4.7670 & -2.9125 & -1.6163 & 0.5313 & 0.2333 & 0.6256 \\
33.3914 & -7.8619 & -38.9117 & 12.5980 & 3.2824 & 1.1853 & 0.1514 & 17.1651
\end{array}\right] x(k) . }
\end{aligned}
$$




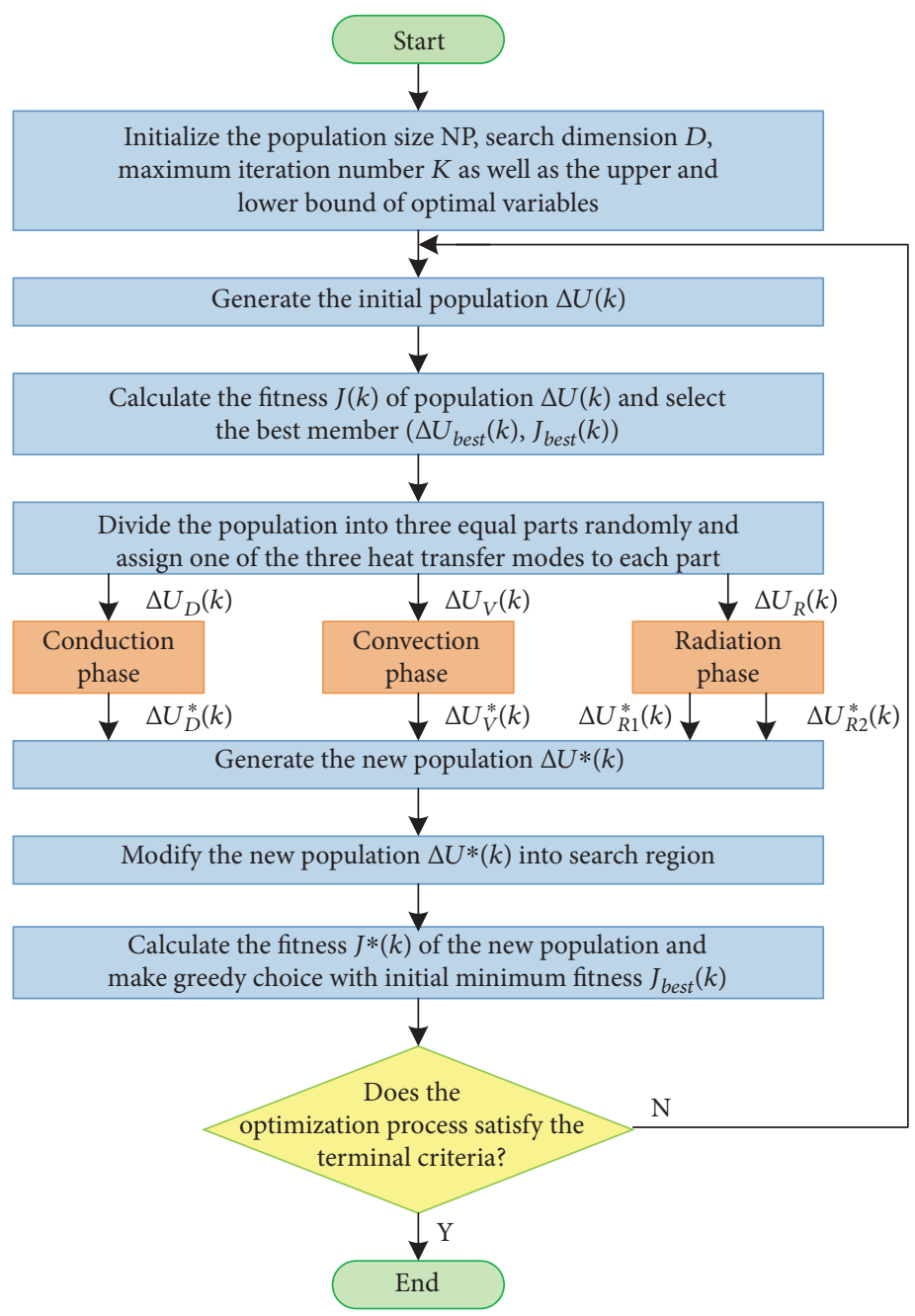

FIGURE 7: Flow chart of the SHTS algorithm.

The verification process is made up of the comparison experiments of tracking performance and disturbance rejection ability with conventional MPC (MPC), fuzzy MPC (FMPC), FMPC with PSO (FMPC + PSO), and FMPC with GWO (FMPC + GWO).

4.1. Parameter Selections. Parameter selection is a significantly important part in the controller design. The empirical method is adopted in this paper because of its easy operation. In addition, some parameters in PSO, GWO, and SHTS can be obtained or fixed automatically through the stochastic technique. Thus, there are only two parameters that need to be determined, namely, the population size NP and maximum iteration number $K$. Considering the fairness of comparison experiment, the corresponding parameters in each algorithm are selected as equal values in every simulation process and listed in Table 2.

What is more, the error weight coefficient vector and control weight coefficient vector of output optimization are set as $Q_{y}=\operatorname{diag}(1,10,1,10)$ and $R_{y}=\operatorname{diag}(0.1,0.1)$, respectively; meanwhile, the state weight coefficient vector and control weight coefficient vector of state optimization are
TABLE 2: Parameter selection results of the control process.

\begin{tabular}{lcccccc}
\hline Parameter & $q$ & $P$ & $M$ & NP & $D$ & $K$ \\
\hline Value & 8 & 20 & 10 & 39 & 20 & 12
\end{tabular}

set as $Q_{x}=\operatorname{diag}(1,1,1,1,1,1,1,1,0.1,1,1,0.1)$ and $R_{x}=\operatorname{diag}$ $(1,1)$, respectively. The lower limitations of $U(k)$ and $\Delta U(k)$ are set as $U_{\min }=[60.5,22.8]^{T}$ and $\Delta U_{\min }=[-0.12$, $-0.18]^{T}$, respectively; at the same time, their upper limitations are set as $U_{\max }=[70.5,28.6]^{T}$ and $\Delta U_{\max }=[0.36$, $0.25]^{T}$, respectively.

4.2. Simulation Results. This part is made up of the tracking performance test and disturbance rejection verification of the proposed controller. At first, based on the selected parameters, the following simulation experiment is executed to test the tracking performance of the controller.

The initial stable operating point of the gas turbine system, in other words, the output variable vector of the controlled system, is set as $[180.79,3000,642.27,1106.8]^{T}$. Then, the set point of the system is changed to $[164.98,3000,652.7,1040.8]^{T}$ at $20 \mathrm{~s}$; the responses of the 

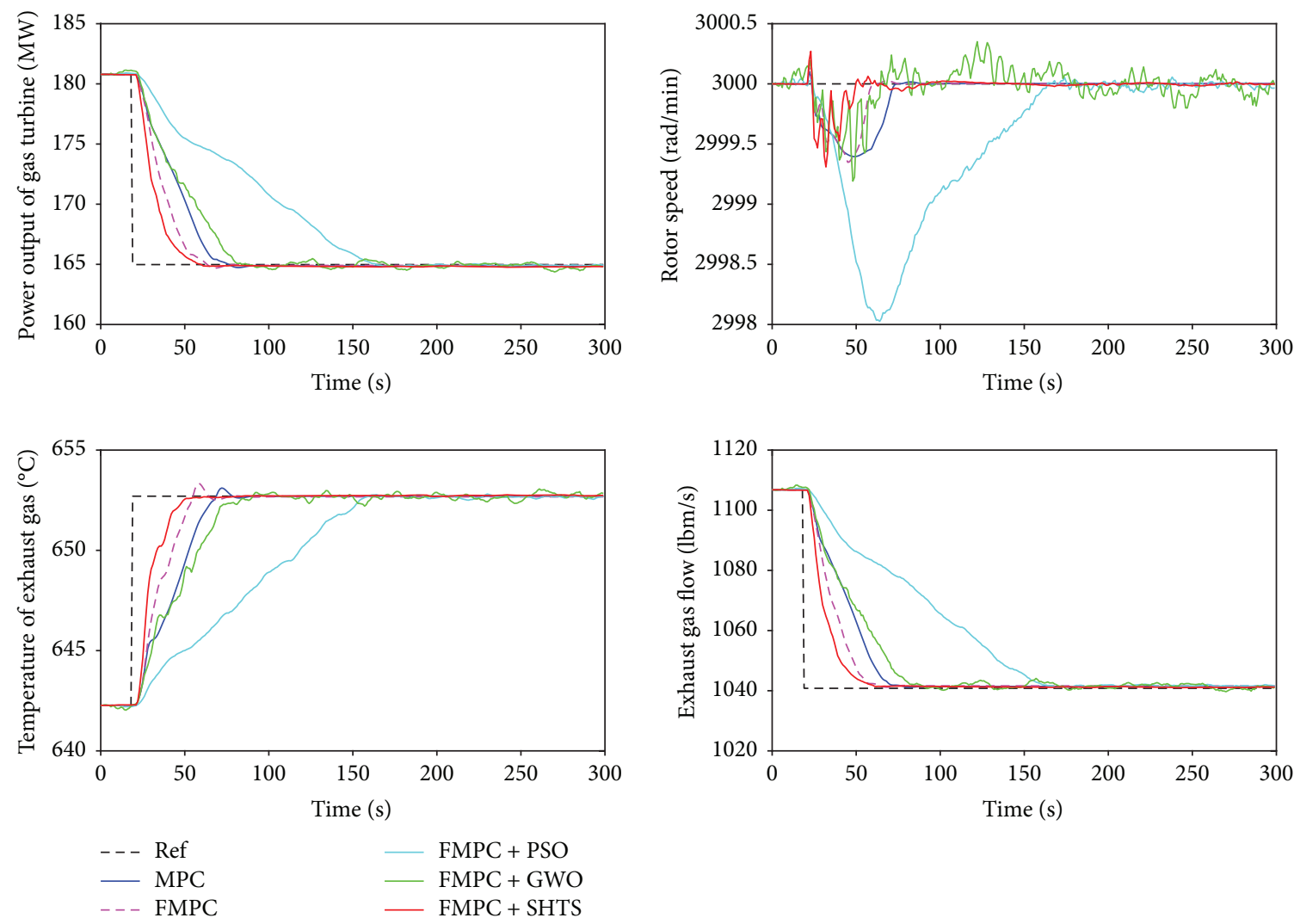

FIGURE 8: Responses of output variables when the set point has changed.
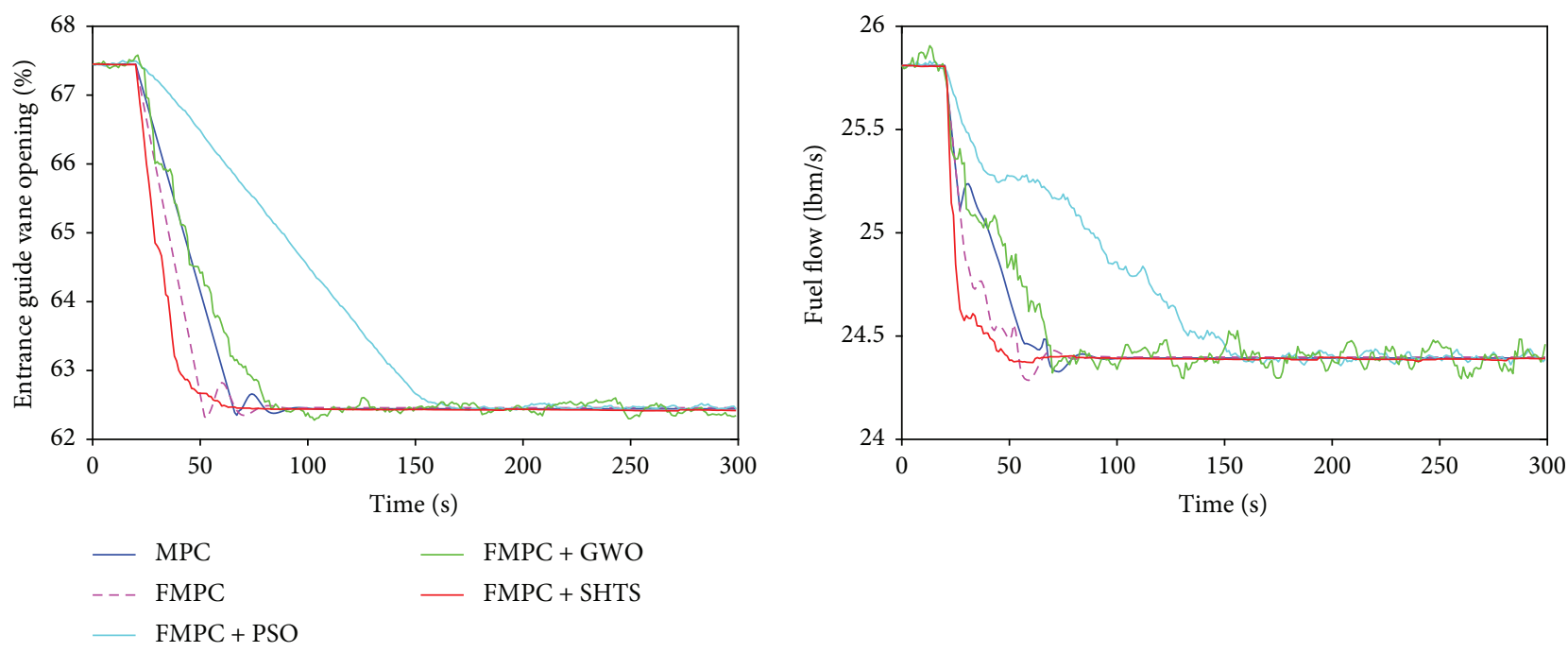

FIGURE 9: Responses of input variables when the set point has changed.

system output variables, input variables, and corresponding state variables are shown as Figures $8-10$, respectively.

In Figures 8 and 9, the black dotted lines present the reference trajectories of the system and the blue solid lines stand for responses of variables controlled by MPC while the magenta dotted lines are the responses of variables controlled by the FMPC. Besides, the cyan solid lines, green solid lines, and red solid lines present the responses of variables controlled by the FMPC which are combined with PSO, GWO, and SHTS, respectively. It is clear from these two figures that when the set point of the system has changed, the variables of the system controlled by the presented algorithm show high tracking performance with lower overshoot and shorter settling time compared with other 

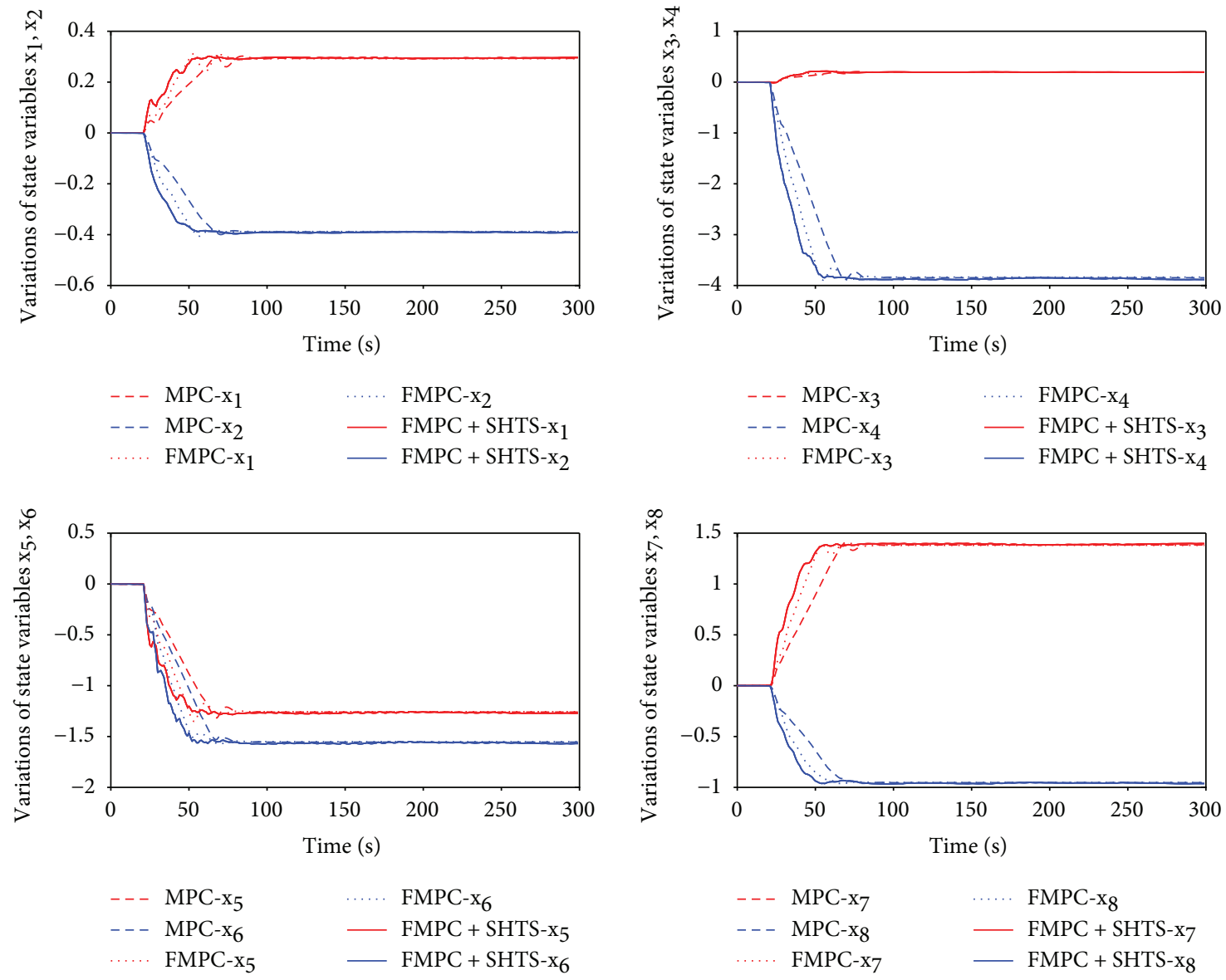

Figure 10: Responses of state variables when the set point has changed.

methods. On account of the state stability of the proposed algorithm, the state variable responses of the system controlled by MPC-, FMPC-, and SHTS-based FMPC are selected for comparison and illustrated in Figure 10. $x_{1}, x_{2}$, $\ldots, x_{8}$ stand for eight state variables of the system. Although not all of the used control methods are included in Figure 10, it is enough for the state stability verification. Because the number of state variables in this gas turbine system is set as 8; therefore, Figure 10 consists of four subfigures and each subfigure obtains two state variables of the system controlled by the aforementioned three strategies. Moreover, the dot-dash lines, dotted lines, and solid lines stand for the state variables of the system controlled by MPC, FMPC, and FMPC which is combined the SHTS (FMPC + SHTS), respectively. From Figure 10, it is obvious that the responses of state variables of the system controlled by the presented algorithm illustrate smoother trends and reach a new steady state in a shorter period of time. Thus, the high state stability of the proposed control method is demonstrated through this comparative experiment.

Apart from tracking performance, the disturbance rejection ability is also an indispensable index to judge the performance of a controller. As a result, the following disturbance rejection experiments are finished for the further performance verification of the adopted algorithm. (i) Apply a negative step signal with amplitude of 5 to the power output of the system while keeping the other three output variables unchanged. Then, the responses of the output variables and input variables are shown in Figure 11.

(ii) Apply a negative step signal with amplitude of 1 to the rotor speed of the system while keeping the other three output variables unchanged. The responses of the output variables and input variables are shown in Figure 12.

(iii) Apply a negative step signal with amplitude of 10 to the temperature of the exhaust gas while keeping the other three output variables unchanged. Then, the responses of the output variables and input variables are depicted in Figure 13.

(iv) Apply a negative step signal with amplitude of 50 to the exhaust gas flow of the system while keeping the other three output variables unchanged. The responses of the output variables and input variables are shown in Figure 14.

It is very clear from Figures 11-14 that the output variables of the system controlled by the presented control method have the smallest overshoot, and the three other 

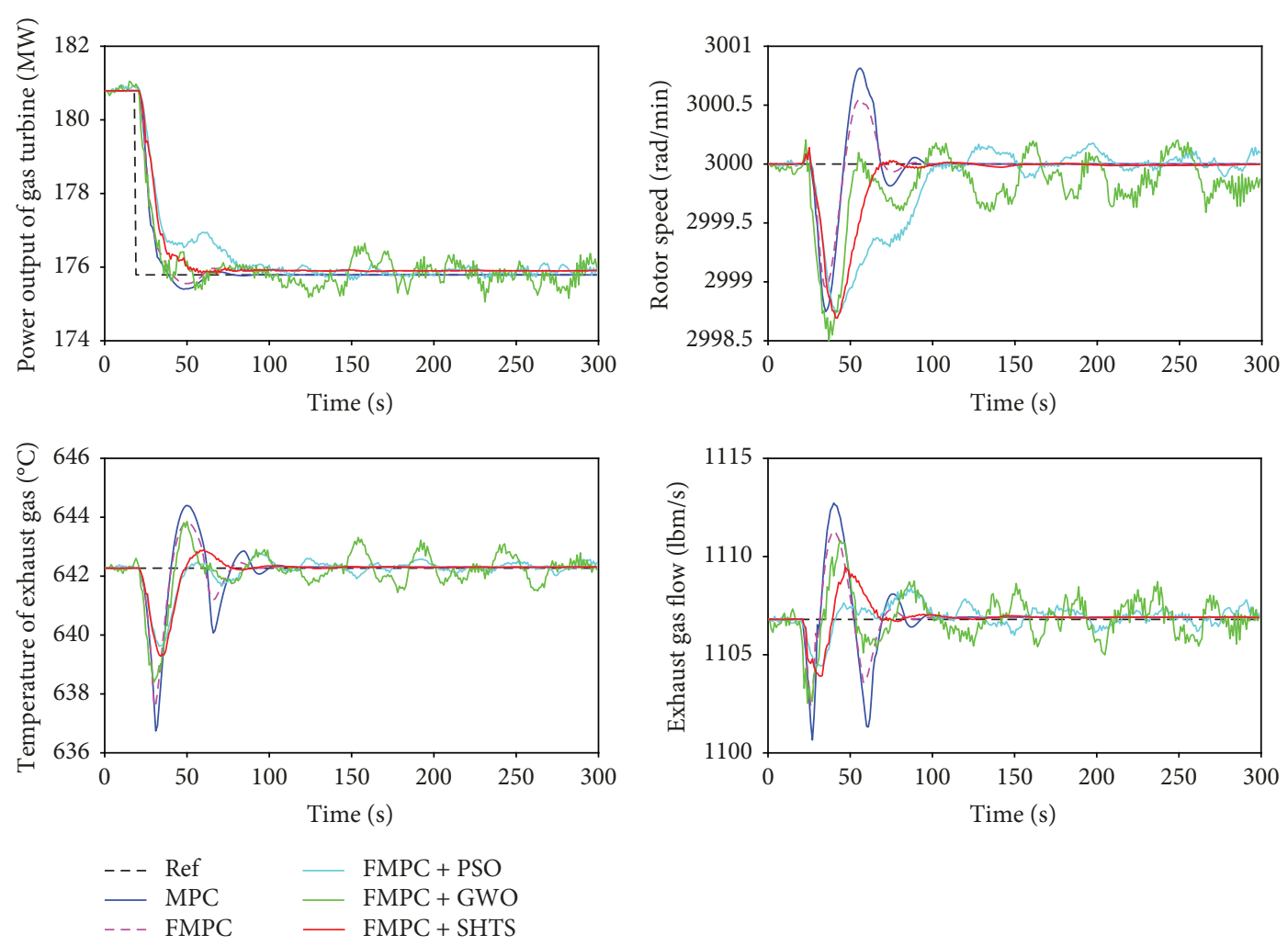

(a)
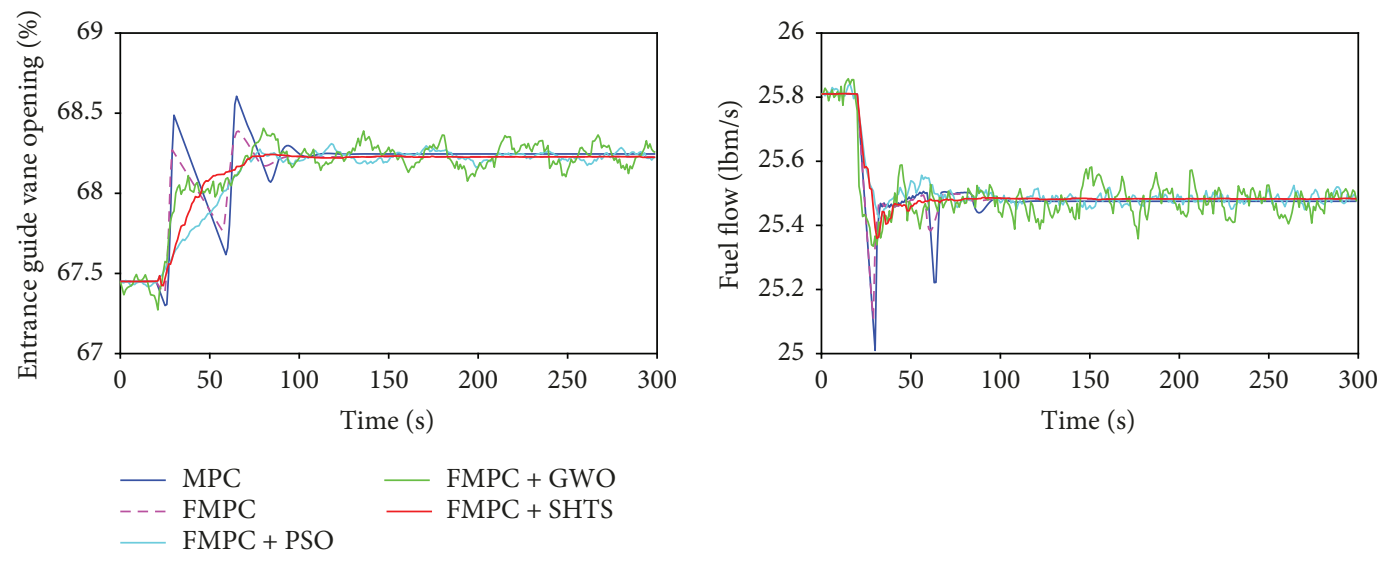

(b)

FIGURE 11: Responses of (a) output variables and (b) input variables when a negative step signal with amplitude of 5 is applied to the power output of the system.

output variables except for the output variable with disturbance can return to the original stable states over the shortest period of time. What is more, the responses of input variables also illustrate the stronger disturbance rejection ability of the controller. It is worth mentioning that the FMPC which is combined with GWO also has the highest settling speed among these five algorithms; however, there are high stable errors existing in the input and output variables which would not be ignored in the control process. Therefore, the presented algorithm is proved to be the best one with remarkable high control performance compared with the other four control methods.
In order to verify the rapidity of the rolling optimization process of the proposed algorithm, the optimization times of above five control strategies in tracking performance tests and disturbance rejection experiments are recorded and listed in following table for comparison.

As is shown in Table 3, the optimization times of MPC are smallest among all of the used controllers because of the absence of the fuzzy selection mechanism. Although the fuzzy selections of the weight coefficients in the objective function have decreased the optimization speed, the higher control performance of FMPC with lower overshoot and shorter settling time as well as higher accuracy has exhibited 

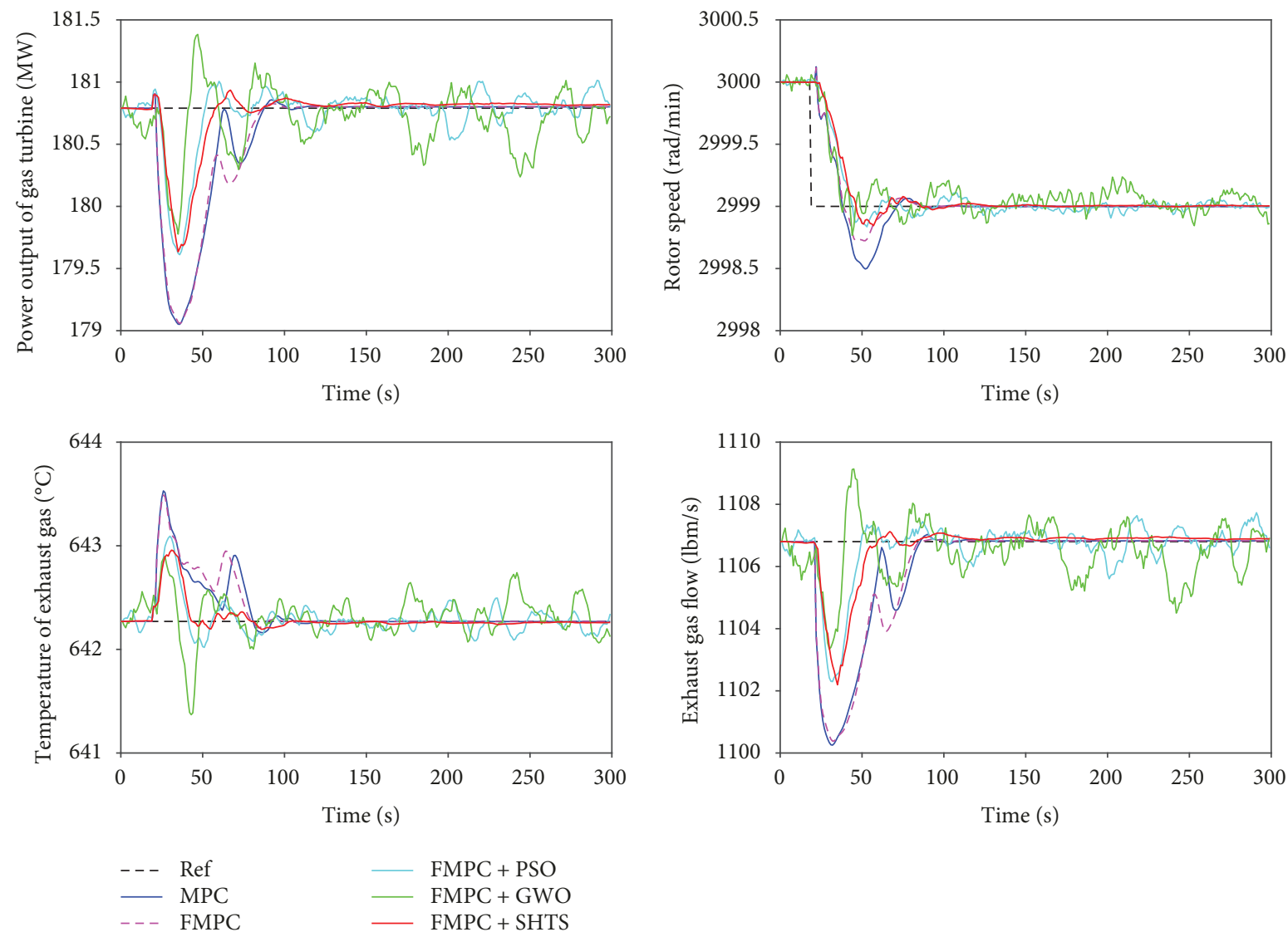

(a)
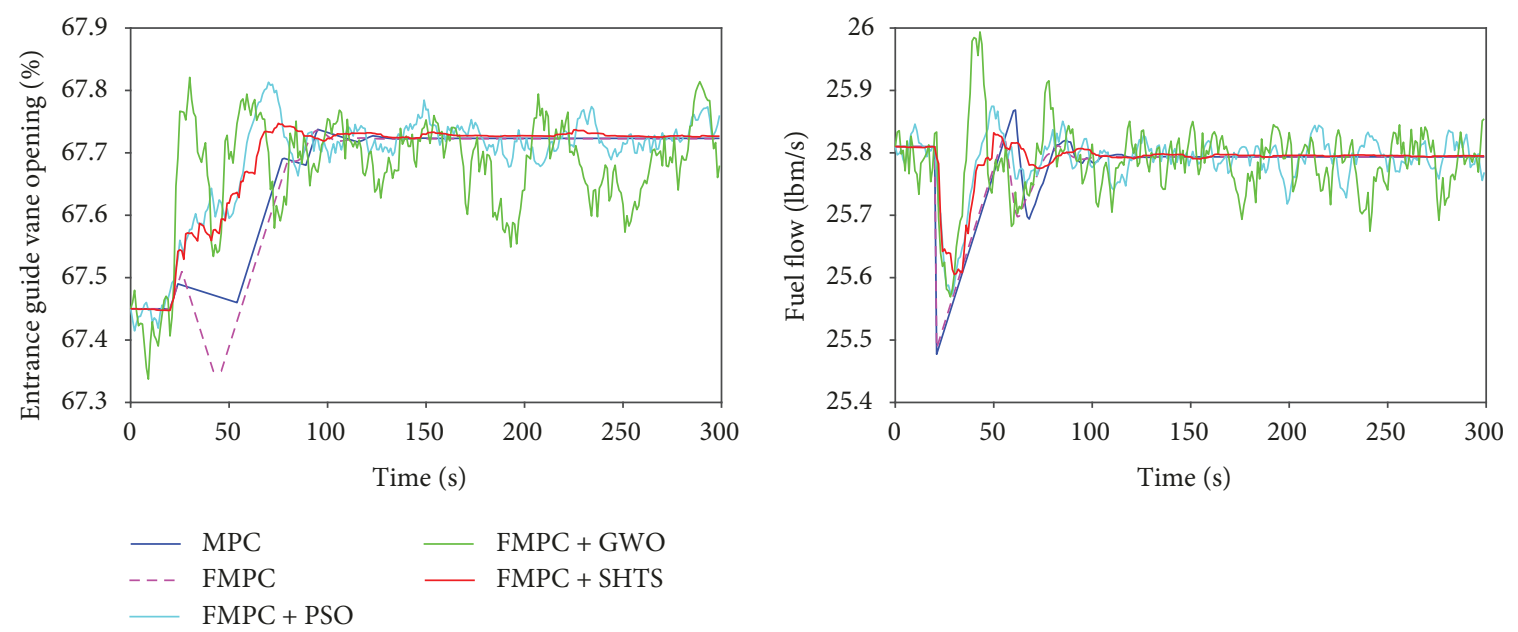

(b)

FIGURE 12: Responses of (a) output variables and (b) input variables when a negative step signal with amplitude of 1 is applied to the rotor speed of the system.

its remarkable superiority compared with traditional MPC. Furthermore, aiming at promoting the optimization speed of FMPC while maintaining its priorities, the quadratic programming solving process of the initial FMPC is replaced by a swarm-based optimization method such as PSO, GWO, and SHTS listed in this table. It is obvious that both the FMPC + GWO and the FMPC + SHTS have shorter periods of optimization time compared with FMPC and FMPC + $\mathrm{PSO}$, whereas the control process of FMPC $+\mathrm{GWO}$ is a poor regulation process with high steady state errors. Thus, the proposed improved FMPC which is combined with SHTS has demonstrated the shortest optimization time, the highest tracking performance, and stronger disturbance rejection ability simultaneously. 

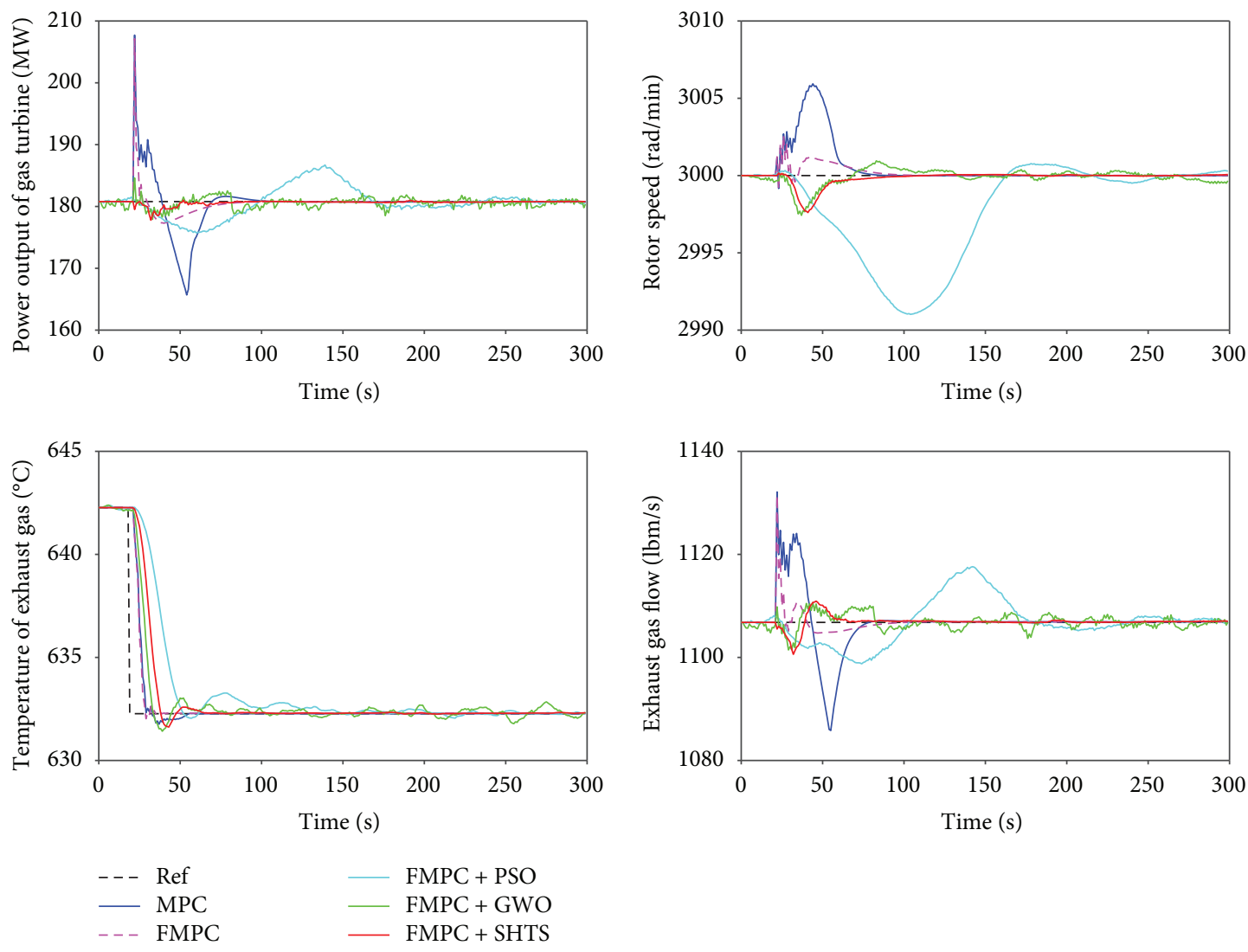

(a)
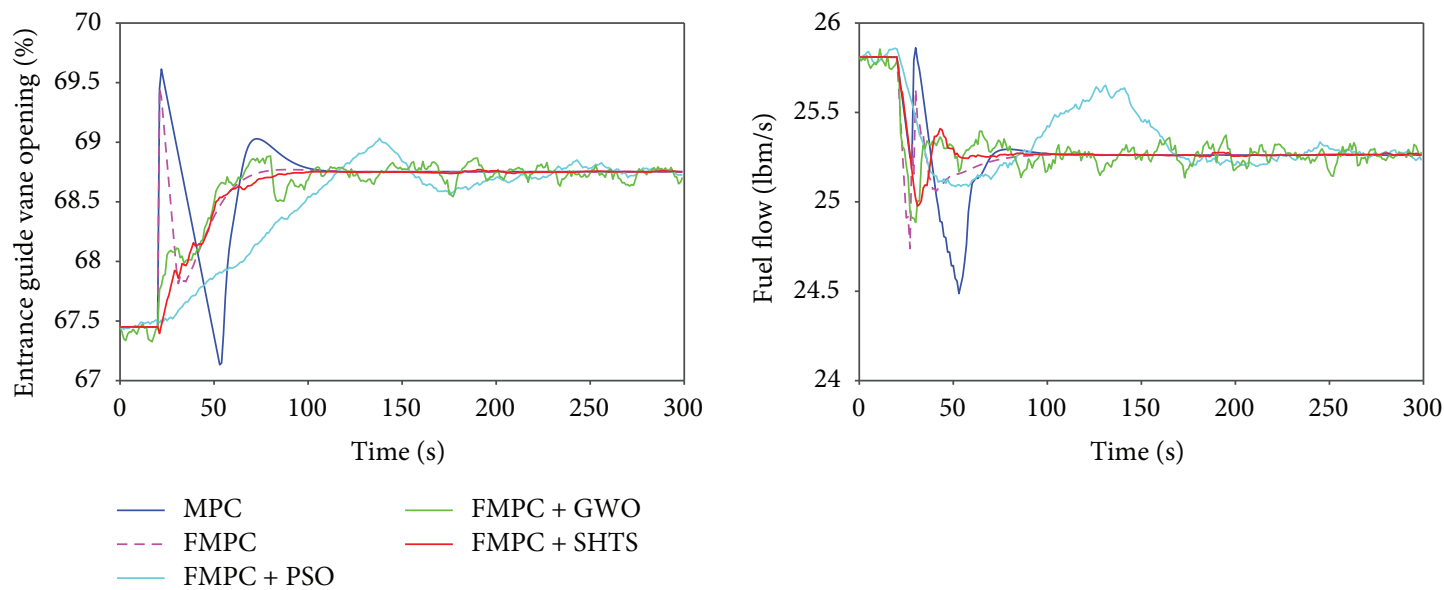

(b)

FIGURE 13: Responses of (a) output variables and (b) input variables when a negative step signal with amplitude of 10 is applied to the temperature of the exhaust gas.

\section{Conclusions}

A novel FMPC algorithm which combined fuzzy selection mechanism and the SHTS while considering the variable constraints is put forward in this paper. Initially, a new objective function is constructed based on the comprehensive consideration of the state optimization problem and output optimization problem. Then, in view of the weight coefficient selections of these two parts in the objective function, a fuzzy selection mechanism is established to deal with the uncertainties existing in the control process and improve the control precision of the controller. Next, in order to promote the optimization speed for the compensation of time increase caused by the fuzzy selection process, the SHTS is adopted in the rolling optimization of FMPC as a replacement of the initial quadratic programming method. Furthermore, the proposed novel FMPC strategy is applied to the gas turbine system of a combined cycle unit in a power plant. The 

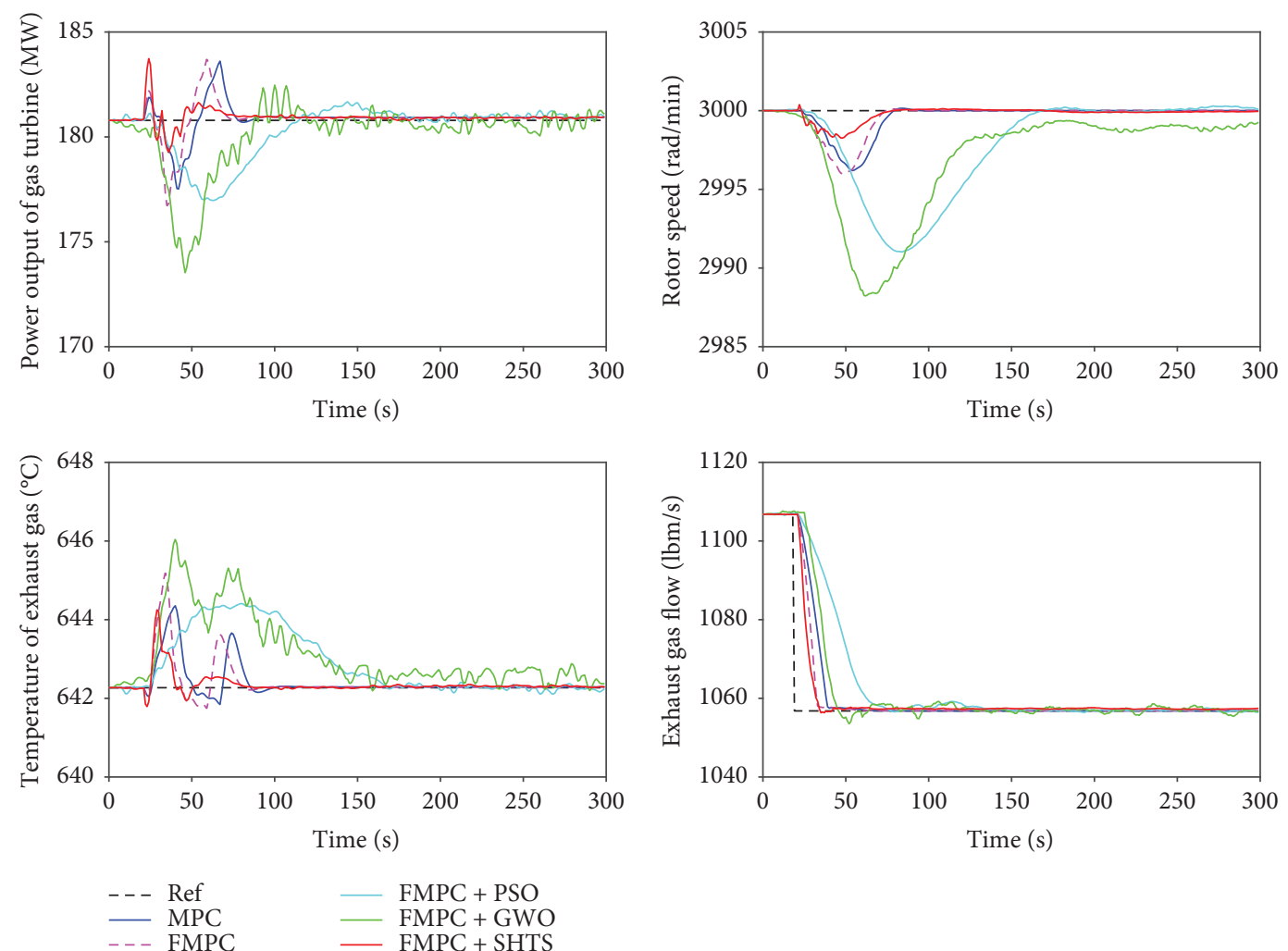

(a)
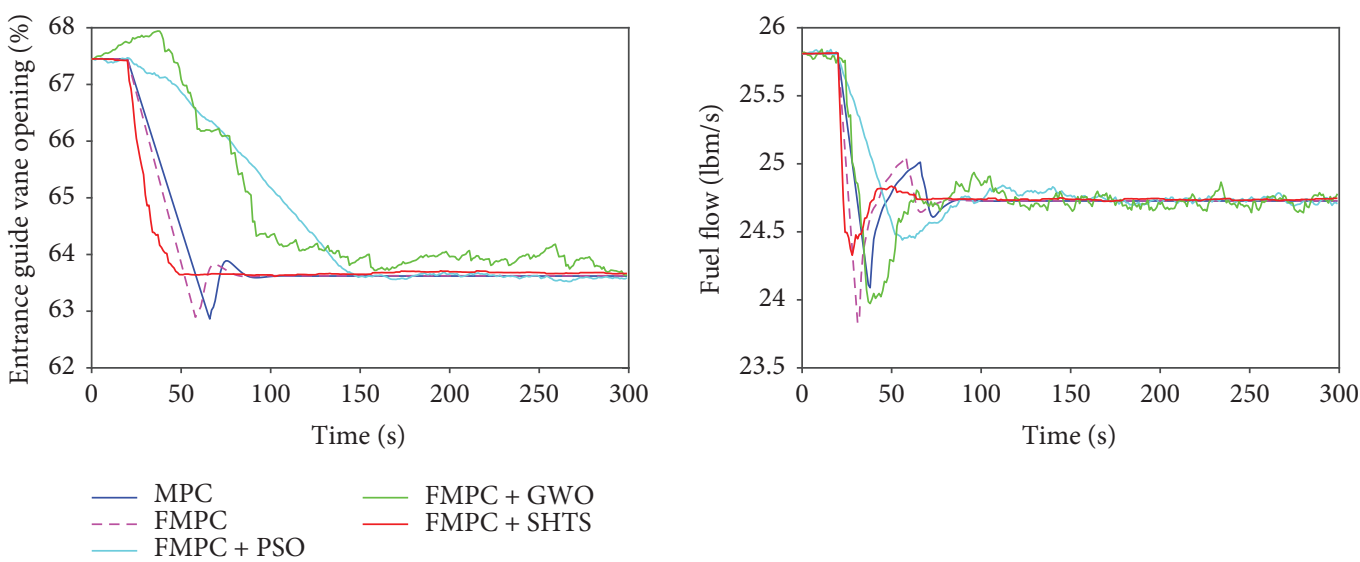

(b)

FIGURE 14: Responses of (a) output variables and (b) input variables when a negative step signal with amplitude of 50 is applied to the exhaust gas flow of the system.

TABLE 3: Optimization times of tracking performance tests and disturbance rejection experiments.

\begin{tabular}{lccccc}
\hline Algorithm & MPC & FMPC & FMPC + PSO & FMPC + GWO & FMPC + SHTS \\
\hline Tracking performance test (s) & 3.7687 & 4.8732 & 10.6927 & 4.7982 & 4.1245 \\
Apply disturbance to $N_{\text {op }}(\mathrm{s})$ & 3.3575 & 5.3149 & 10.2229 & 4.7277 & 4.1673 \\
Apply disturbance to $N(\mathrm{~s})$ & 3.9810 & 4.9629 & 10.4244 & 4.7782 & 4.1007 \\
Apply disturbance to $T_{\text {ex }}(\mathrm{s})$ & 3.0809 & 4.9979 & 10.7982 & 4.9096 & 4.3660 \\
Apply disturbance to $G_{\text {ex }}$ (s) & 3.0049 & 4.6834 & 11.0637 & 4.9561 & 4.3375 \\
\hline
\end{tabular}


simulation results demonstrate its remarkable advantages with higher control accuracy, stronger disturbance rejection ability, and less optimization time.

\section{Data Availability}

The identified state space model of the gas turbine system in a combined cycle power plant which was used to support the findings of this study is included within the article. However, we are so sorry that the actual operating data of the power plant used to identify the gas turbine system model is unavailable due to the confidentiality requirement of Taiyanggong power plant. Furthermore, the parameter selections of the control algorithms are described in the manuscript.

\section{Conflicts of Interest}

The authors declare that they have no conflicts of interest.

\section{Acknowledgments}

This work is supported by the Fundamental Research Funds for the Central Universities (2016ZZD03), the National Science Foundation of China (61603136), and the Colleges and Universities Key Laboratory of Intelligent Integrated Automation (Intelligent Automation 201502). The reviewers' instructive and valuable suggestions in improving the quality of the paper are extremely appreciated.

\section{References}

[1] D. Wang, Y. Zhou, and X. Li, “A dynamic model used for controller design for fast cut back of coal-fired boiler-turbine plant," Energy, vol. 144, pp. 526-534, 2018.

[2] G. Hou, Y. Yang, Z. Jiang, Q. Li, and J. Zhang, "A new approach of modeling an ultra-super-critical power plant for performance improvement," Energies, vol. 9, no. 5, p. 310, 2016.

[3] Z. Yang, K. Li, Q. Niu, and Y. Xue, "A comprehensive study of economic unit commitment of power systems integrating various renewable generations and plug-in electric vehicles," Energy Conversion and Management, vol. 132, pp. 460-481, 2017.

[4] X. Wang, C. Yang, M. Huang, and X. Ma, "Multi-objective optimization of a gas turbine-based CCHP combined with solar and compressed air energy storage system," Energy Conversion and Management, vol. 164, pp. 93-101, 2018.

[5] J. D. Wojcik and J. Wang, "Feasibility study of combined cycle gas turbine (CCGT) power plant integration with adiabatic compressed air energy storage (ACAES)," Applied Energy, vol. 221, pp. 477-489, 2018.

[6] T. Liu, G. Zhang, Y. Li, and Y. Yang, "Performance analysis of partially recuperative gas turbine combined cycle under offdesign conditions," Energy Conversion and Management, vol. 162, pp. 55-65, 2018.

[7] M. A. Elhosseini, R. A. El Sehiemy, A. H. Salah, and M. A. Abido, "Modeling and control of an interconnected combined cycle gas turbine using fuzzy and ANFIS controllers," Electrical Engineering, vol. 100, no. 2, pp. 763-785, 2018.
[8] L. Herraiz, E. S. Fernandez, E. Palfi, and M. Lucquiaud, "Selective exhaust gas recirculation in combined cycle gas turbine power plants with post-combustion $\mathrm{CO}_{2}$ capture," International Journal of Greenhouse Gas Control, vol. 71, pp. 303-321, 2018.

[9] J. M. L. Domínguez and T. J. M. Sanguino, "Design, modelling, and implementation of a fuzzy controller for an intelligent road signaling system," Complexity, vol. 2018, Article ID 1849527, 14 pages, 2018.

[10] Y. Ma, P. Duan, Y. Sun, and H. Chen, "Equalization of lithiumion battery pack based on fuzzy logic control in electric vehicle," IEEE Transactions on Industrial Electronics, vol. 65, no. 8, pp. 6762-6771, 2018.

[11] X.-W. Chen and Y. Zhou, "Modelling and analysis of automobile vibration system based on fuzzy theory under different road excitation information," Complexity, vol. 2018, Article ID 2381568, 9 pages, 2018.

[12] Y. Zheng, J. Zhou, Y. Xu, Y. Zhang, and Z. Qian, “A distributed model predictive control based load frequency control scheme for multi-area interconnected power system using discretetime Laguerre functions," ISA Transactions, vol. 68, pp. 127140, 2017.

[13] A. Taieb, M. Soltani, and A. Chaari, "Parameter optimization of MIMO fuzzy optimal model predictive control by APSO," Complexity, vol. 2017, Article ID 5813192, 11 pages, 2017.

[14] H. Gouta, S. H. Saïd, N. Barhoumi, and F. M'Sahli, "Generalized predictive control for a coupled four tank MIMO system using a continuous-discrete time observer," ISA Transactions, vol. 67, pp. 280-292, 2017.

[15] X. Feng, S. Huo, J. Zhang, and H. Shen, "Fuzzy predictive temperature control for a class of metallurgy lime kiln models," Complexity, vol. 21, no. S2, 258 pages, 2016.

[16] C. Yang, J. Na, G. Li, Y. Li, and J. Zhong, "Neural network for complex systems: theory and applications," Complexity, vol. 2018, Article ID 3141805, 2 pages, 2018.

[17] B. Bayar and M. C. Stamm, "Constrained convolutional neural networks: a new approach towards general purpose image manipulation detection," IEEE Transactions on Information Forensics and Security, vol. 13, no. 11, pp. 26912706, 2018.

[18] W. Liu, Z. Yang, and K. Bi, "Forecasting the acquisition of university spin-outs: an RBF neural network approach," Complexity, vol. 2017, Article ID 6920904, 8 pages, 2017.

[19] S. Sotirov, E. Sotirova, V. Atanassova et al., "A hybrid approach for modular neural network design using intercriteria analysis and intuitionistic fuzzy logic," Complexity, vol. 2018, Article ID 3927951, 11 pages, 2018.

[20] I. Nielsen and D. Axehill, "Low-rank modifications of Riccati factorizations for model predictive control," IEEE Transactions on Automatic Control, vol. 63, no. 3, pp. 872-879, 2018.

[21] T. M. Pinho, J. P. Coelho, G. Veiga, A. P. Moreira, and J. Boaventura-Cunha, "A multilayer model predictive control methodology applied to a biomass supply chain operational level," Complexity, vol. 2017, Article ID 5402896, 10 pages, 2017.

[22] J. S. Hernandez, R. Rivas-Perez, and J. J. S. Moriano, "Design of a generalized predictive controller for temperature control in a cement rotary kiln," IEEE Latin America Transactions, vol. 16, no. 4, pp. 1015-1021, 2018.

[23] X. Liu, C. Zhang, K. Li, and Q. Zhang, "Robust current control-based generalized predictive control with sliding 
mode disturbance compensation for PMSM drives," ISA Transactions, vol. 71, Part 2, pp. 542-552, 2017.

[24] A. Alessandretti, A. P. Aguiar, and C. N. Jones, "An input-tostate-stability approach to economic optimization in model predictive control," IEEE Transactions on Automatic Control, vol. 62, no. 12, pp. 6081-6093, 2017.

[25] Z. Tian, J. Yuan, X. Zhang, L. Kong, and J. Wang, "Modeling and sliding mode predictive control of the ultra-supercritical boiler-turbine system with uncertainties and input constraints," ISA Transactions, vol. 76, pp. 43-56, 2018.

[26] T. Zou, S. Wu, and R. Zhang, "Improved state space model predictive fault-tolerant control for injection molding batch processes with partial actuator faults using GA optimization," ISA Transactions, vol. 73, pp. 147-153, 2018.

[27] L. Faes, A. Porta, M. Javorka, and G. Nollo, "Efficient computation of multiscale entropy over short biomedical time series based on linear state-space models," Complexity, vol. 2017, Article ID 1768264, 13 pages, 2017.

[28] S. El-Ferik, B. A. Siddiqui, and F. L. Lewis, "Distributed nonlinear MPC of multi-agent systems with data compression and random delays," IEEE Transactions on Automatic Control, vol. 61, no. 3, pp. 817-822, 2016.

[29] S. Zhang and W. Mao, "Optimal operation of coal conveying systems assembled with crushers using model predictive control methodology," Applied Energy, vol. 198, pp. 65-76, 2017.

[30] J. Tao, L. Ma, and Y. Zhu, "Improved control using extended non-minimal state space MPC and modified LQR for a kind of nonlinear systems," ISA Transactions, vol. 65, pp. 319326, 2016.

[31] L. Dai, Y. Xia, Y. Gao, and M. Cannon, "Distributed stochastic MPC of linear systems with additive uncertainty and coupled probabilistic constraints," IEEE Transactions on Automatic Control, vol. 62, no. 7, pp. 3474-3481, 2017.

[32] L. Dai, Y. Xia, Y. Gao, and M. Cannon, "Distributed stochastic MPC for systems with parameter uncertainty and disturbances," International Journal of Robust and Nonlinear Control, vol. 28, no. 6, pp. 2424-2441, 2018.

[33] S. Wang, L. Zhang, X. Sun, and H. Jia, "Inversion of thermal conductivity in two-dimensional unsteady-state heat transfer system based on boundary element method and decentralized fuzzy inference," Complexity, vol. 2018, Article ID 8783946, 9 pages, 2018.

[34] Y. Fan, K. Xing, and X. Jiang, "Fuzzy adaptation algorithms' control for robot manipulators with uncertainty modelling errors," Complexity, vol. 2018, Article ID 5468090, 8 pages, 2018.

[35] R. Osorio, J. M. Alonso, N. Vazquez et al., "Fuzzy logic control with an improved algorithm for integrated LED drivers," IEEE Transactions on Industrial Electronics, vol. 65, no. 9, pp. 69947003, 2018.

[36] N. Bouzera, M. Oussalah, N. Mezhoud, and A. Khireddine, "Fuzzy extended Kalman filter for dynamic mobile localization in urban area using wireless network," Applied Soft Computing, vol. 57, pp. 452-467, 2017.

[37] N. Gao, Q. Li, and X. Zhou, "Fuzzy extended filters on residuated lattices," Soft Computing, vol. 22, no. 7, pp. 2321-2328, 2018.

[38] I. Boulkaibet, K. Belarbi, S. Bououden, T. Marwala, and M. Chadli, "A new T-S fuzzy model predictive control for nonlinear processes," Expert Systems with Applications, vol. 88, pp. 132-151, 2017.
[39] L. Dalhoumi, M. Chtourou, and M. Djemel, "On the fuzzy model predictive control of interconnected nonlinear systems," Arabian Journal for Science and Engineering, vol. 42, no. 7, pp. 2759-2776, 2017.

[40] F. Zhang, X. Wu, and J. Shen, "Extended state observer based fuzzy model predictive control for ultra-supercritical boilerturbine unit," Applied Thermal Engineering, vol. 118, pp. 90$100,2017$.

[41] L. Liu, Y. Wang, F. Xie, and J. Gao, "Legendre cooperative PSO strategies for trajectory optimization," Complexity, vol. 2018, Article ID 5036791, 13 pages, 2018.

[42] O. Abedinia, A. Ghasemi, and N. Ojaroudi, "Improved time varying inertia weight PSO for solved economic load dispatch with subsidies and wind power effects," Complexity, vol. 21, no. 4, 49 pages, 2016.

[43] W.-D. Chang, "A modified PSO algorithm for IIR digital filter modeling," Journal of Circuits Systems and Computers, vol. 27, no. 5, article 1850073, 2018.

[44] C. B. Kalayci, O. Ertenlice, H. Akyer, and H. Aygoren, "An artificial bee colony algorithm with feasibility enforcement and infeasibility toleration procedures for cardinality constrained portfolio optimization," Expert Systems with Applications, vol. 85, pp. 61-75, 2017.

[45] H. Liu, H. Wu, and Y. Li, "Smart wind speed forecasting using EWT decomposition, GWO evolutionary optimization, RELM learning and IEWT reconstruction," Energy Conversion and Management, vol. 161, pp. 266-283, 2018.

[46] V. K. Patel and V. J. Savsani, "Heat transfer search (HTS): a novel optimization algorithm," Information Sciences, vol. 324, pp. 217-246, 2015.

[47] D. Maharana and P. Kotecha, "Simultaneous heat transfer search for computationally expensive numerical optimization," in 2016 IEEE Congress on Evolutionary Computation (CEC), pp. 2982-2988, Vancouver, BC, Canada, July 2016, IEEE.

[48] G. Hou, H. Du, Y. Yang, C. Huang, and J. Zhang, "Coordinated control system modelling of ultra-supercritical unit based on a new T-S fuzzy structure," ISA Transactions, vol. 74, pp. 120$133,2018$. 


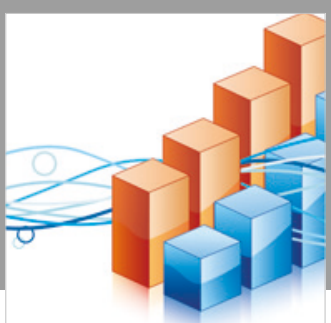

Advances in

Operations Research

\section{-n-m}
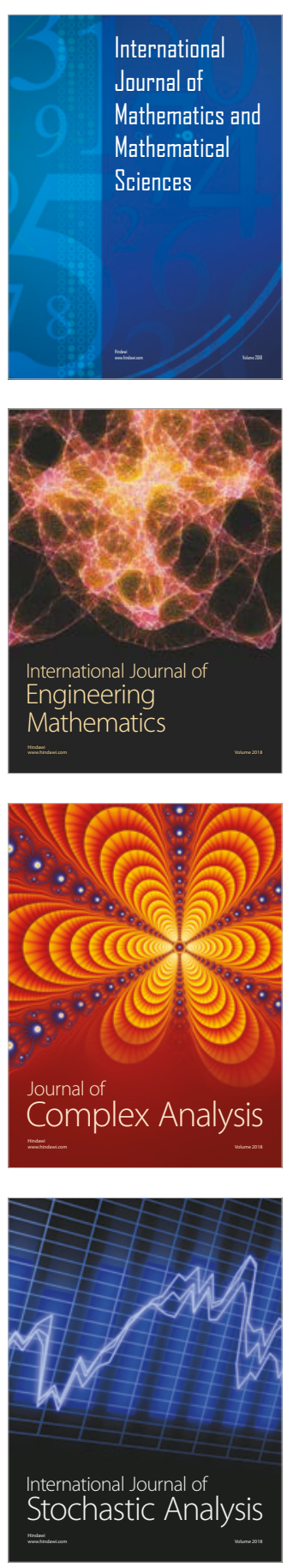
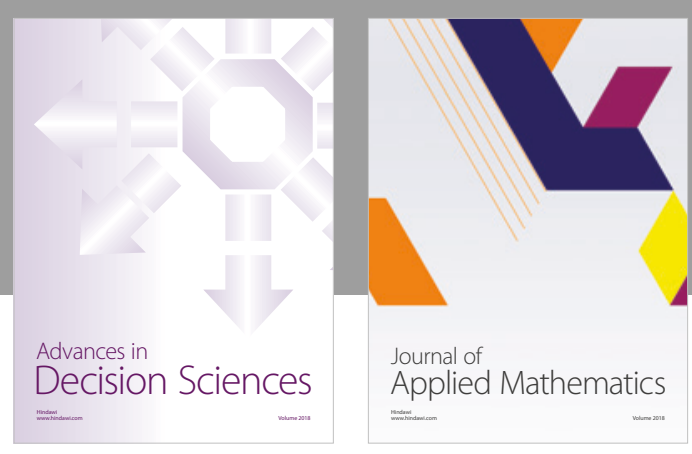

Journal of

Applied Mathematics
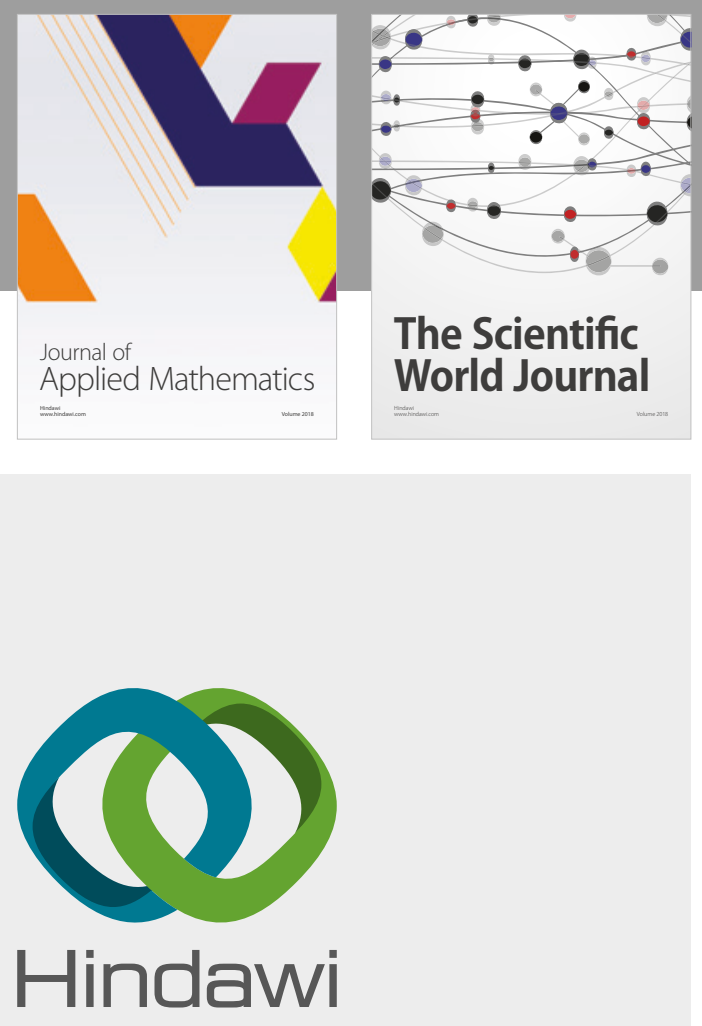

Submit your manuscripts at

www.hindawi.com

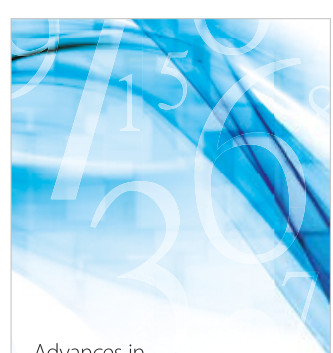

Advances in
Numerical Analysis
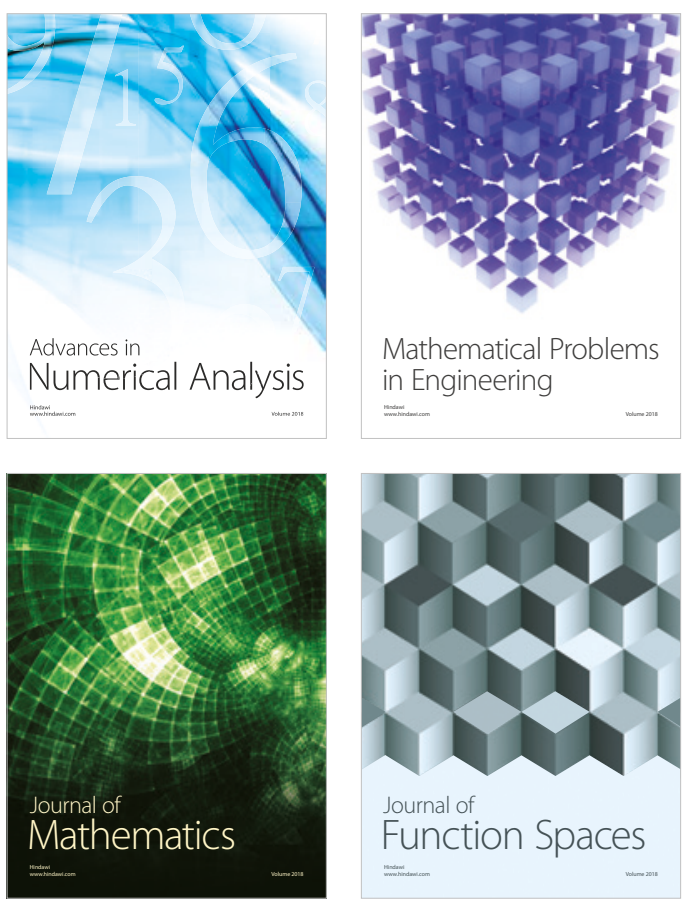

Mathematical Problems in Engineering

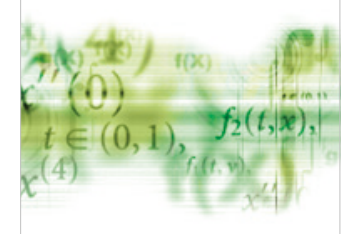

International Journal of

Differential Equations

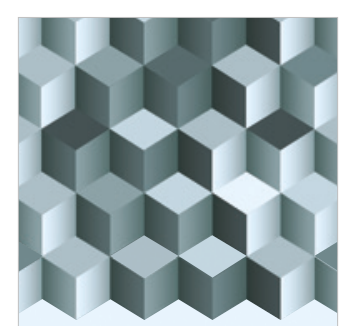

Journal of

Function Spaces
The Scientific

World Journal

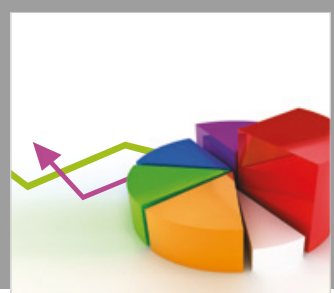

Journal of

Probability and Statistics
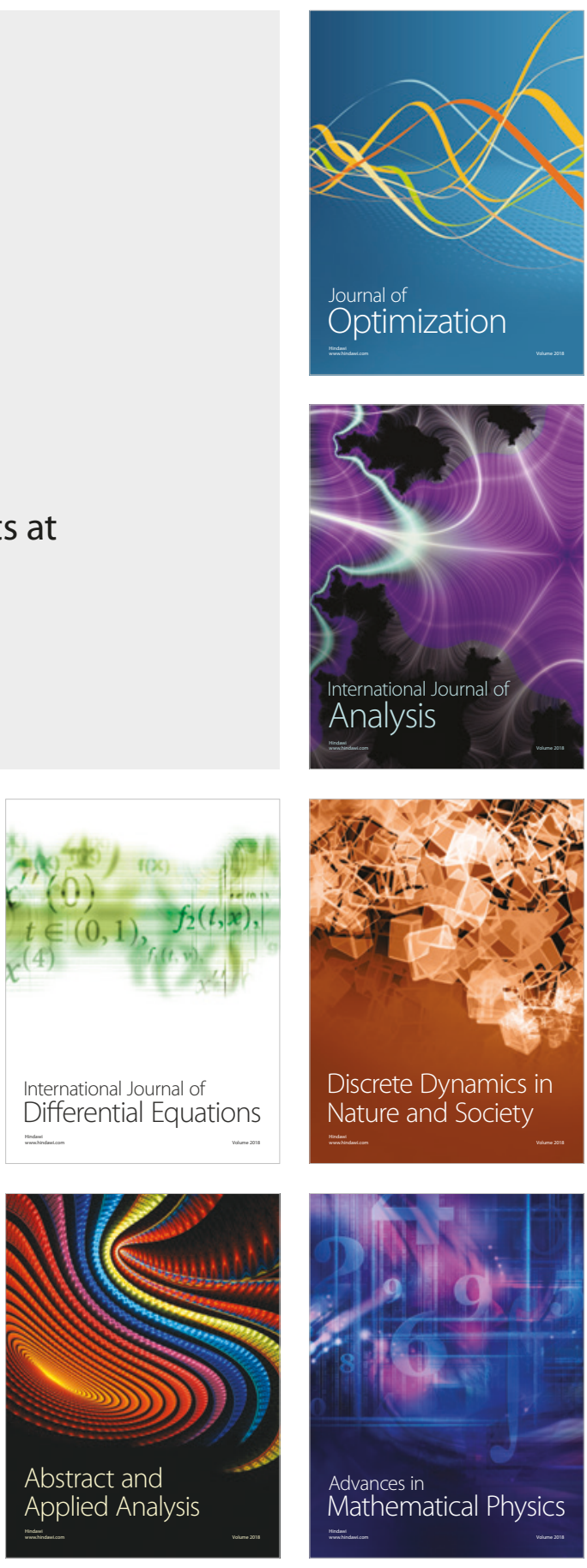